\title{
Asymptotics of reaction-diffusion fronts with one static and one diffusing reactant
}

\author{
Martin Z. Bazant ${ }^{\mathrm{a}, *}$, H.A. Stone ${ }^{\mathrm{b}}$ \\ a Department of Mathematics, Building 2-363B, Massachusetts Institute of Technology, Cambridge, MA 02139, USA \\ ${ }^{\mathrm{b}}$ Division of Engineering and Applied Sciences, Harvard University, Cambridge, MA 02138, USA \\ Received 8 April 1999; received in revised form 14 June 2000; accepted 23 June 2000 \\ Communicated by C.K.R.T. Jones
}

\begin{abstract}
The long-time behavior of a reaction-diffusion front between one static (e.g. porous solid) reactant A and one initially separated diffusing reactant $\mathrm{B}$ is analyzed for the mean-field reaction-rate density $R\left(\rho_{\mathrm{A}}, \rho_{\mathrm{B}}\right)=k \rho_{\mathrm{A}}^{m} \rho_{\mathrm{B}}^{n}$. A uniformly valid asymptotic approximation is constructed from matched self-similar solutions in a "reaction front" (of width $w \sim t^{\alpha}$, where $R \sim t^{\beta}$ enters the dominant balance) and a "diffusion layer" (of width $W \sim t^{1 / 2}$, where $R$ is negligible). The limiting solution exists if and only if $m, n \geq 1$, in which case the scaling exponents are uniquely given by $\alpha=(m-1) / 2(m+1)$ and $\beta=m /(m+1)$. In the diffusion layer, the common ad hoc approximation of neglecting reactions is given mathematical justification, and the exact transient decay of the reaction rate is derived. The physical effects of higher-order kinetics $(m, n>$ 1), such as the broadening of the reaction front and the slowing of transients, are also discussed. () 2000 Elsevier Science B.V. All rights reserved.
\end{abstract}

PACS: $05.40+\mathrm{j}$; 82.20.-w; 02.30.Jr

Keywords: Reaction kinetics; Diffusion; Partial differential equations; Asymptotic analysis; Similarity solutions

\section{Introduction}

In the decade that has passed since the pioneering analytical study of Gálfi and Rácz [1], there has emerged a substantial body of research devoted to experimental [2,3,36-43], computational [4-10,44-49] and analytical [11-16,50] studies of reaction-diffusion systems with two initially separated, diffusing species A and B reacting to produce an inert product $\mathrm{C}$ according to the chemical formula

$$
m^{\prime} \mathrm{A}+n^{\prime} \mathrm{B} \rightarrow \mathrm{C} \text { (inert), }
$$

where $m^{\prime}$ and $n^{\prime}$, the stoichiometric coefficients, are positive integers. Theoretical studies have focused almost exclusively on the "one-dimensional" case of an infinite, flat reaction front between two regions of homogeneous composition of either A or B (see Fig. 1). This idealized situation is believed to capture much of the

\footnotetext{
* Corresponding author. Tel.: +1-617-253-1713; fax: +1-617-253-8911

E-mail address: bazant@math.mit.edu (M.Z. Bazant).
} 

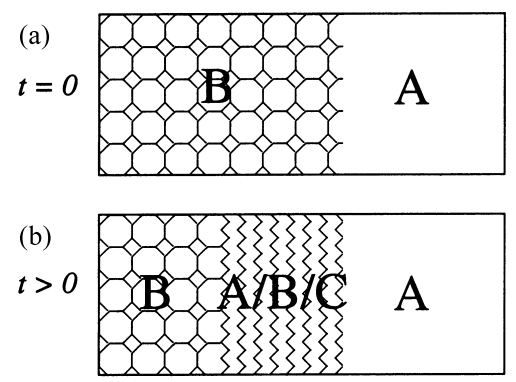

reaction front propagation
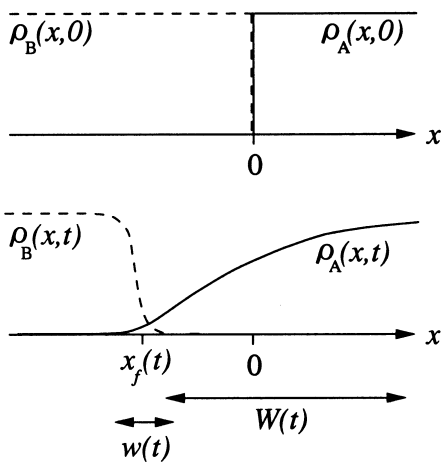

Fig. 1. Schematic diagrams (on the left) and concentration sketches (on the right) showing (a) the diffusing reactant A initially separated from the static reactant B and (b) the formation of a reaction front propagating into the region rich in species B at some later time leaving in its wake a mixture of species A and the reaction product C. Various quantities discussed in Section 3 are also indicated, e.g. the position of the reaction front $x_{\mathrm{f}}(t)$ and the widths of the diffusion layer $W(t)$ and reaction front $w(t)$.

essential physics of reaction fronts commonly observed in various chemical [17,18] and biological [19,20] systems.

The standard continuum model for such a one-dimensional reaction front involves a pair of nonlinear partial differential equations $[1,5,11-13,44-47,50]$ :

$$
\begin{aligned}
& \frac{\partial \rho_{\mathrm{A}}}{\partial T}=D_{\mathrm{A}} \frac{\partial^{2} \rho_{\mathrm{A}}}{\partial X^{2}}-m^{\prime} R\left(\rho_{\mathrm{A}}, \rho_{\mathrm{B}}\right), \\
& \frac{\partial \rho_{\mathrm{B}}}{\partial T}=D_{\mathrm{B}} \frac{\partial^{2} \rho_{\mathrm{B}}}{\partial X^{2}}-n^{\prime} R\left(\rho_{\mathrm{A}}, \rho_{\mathrm{B}}\right),
\end{aligned}
$$

subject to the boundary conditions

$$
\rho_{\mathrm{A}}(-\infty, T)=0, \quad \rho_{\mathrm{A}}(\infty, T)=\rho_{\mathrm{A}}^{0}, \quad \rho_{\mathrm{B}}(-\infty, T)=\rho_{\mathrm{B}}^{0}, \quad \rho_{\mathrm{B}}(\infty, T)=0
$$

and the initial conditions

$$
\rho_{\mathrm{A}}(X, 0)=\rho_{\mathrm{A}}^{0} H(X), \quad \rho_{\mathrm{B}}(X, 0)=\rho_{\mathrm{B}}^{0} H(-X),
$$

where $\rho_{\mathrm{A}}(X, T)$ and $\rho_{\mathrm{B}}(X, T)$ are the concentrations, $D_{\mathrm{A}}$ and $D_{\mathrm{B}}$ are the diffusion coefficients of $\mathrm{A}$ and $\mathrm{B}$, respectively, $\rho_{\mathrm{A}}^{0}>0$ and $\rho_{\mathrm{B}}^{0}>0$ are constants, $H(X)$ is the Heaviside unit step function and $R\left(\rho_{\mathrm{A}}, \rho_{\mathrm{B}}\right)$ is the reaction rate density for production of species $C$. (Note that upper-case letters denote quantities with dimensions, e.g. $X$ and $T$ for space and time, respectively. Lower-case letters for the corresponding dimensionless quantities are introduced in Section 2.1.) The reactants are completely separated at first according to (4), but for $T>0$ they diffuse together and react, which decreases the concentrations wherever $\rho_{\mathrm{A}}(X, T) \rho_{\mathrm{B}}(X, T)>0$. Diffusion acts to replenish any depleted regions. As a result, the system develops a localized, moving region, the "reaction front", where the reaction rate $R\left(\rho_{\mathrm{A}}, \rho_{\mathrm{B}}\right)$ is greatest and which is fed by diffusion from the distant particle reservoirs described by the boundary conditions. The dynamics of this reaction front are described by the long-time asymptotics of the nonlinear initial-boundary-value problem (2a)-(4).

The nonlinear reaction term $R\left(\rho_{\mathrm{A}}, \rho_{\mathrm{B}}\right)$ is usually assumed to have the form of a power law

$$
R\left(\rho_{\mathrm{A}}, \rho_{\mathrm{B}}\right)=k \rho_{\mathrm{A}}^{m} \rho_{\mathrm{B}}^{n},
$$


where $k$ is a rate constant, and $m$ and $n$ are, respectively, the "kinetic orders" of A and B in the reaction [18]. For a one-step reaction with sufficient mixing (see below), $m=m^{\prime}$ and $n=n^{\prime}$, but for more complex, multi-step reactions, $m$ and $n$ are determined by the stoichiometric coefficients of the (often unknown) rate-limiting step. Although $m$ and $n$ are usually taken to be positive integers, non-integer values of $m$ and $n$ can arise in certain situations [18]. We will see that a well-defined reaction front exists for any real numbers $m, n \geq 1$, but not for $m<1$ or $n<1$.

Technically, by assuming in (2a) and (2b) that the reaction rate $R$ depends only on the average local concentrations (and not on any fluctuations or many-body effects), we have made the "mean-field approximation" [21,51]. In low-dimensional systems, such as ion channels $(d=1)$ or catalytic surfaces $(d=2)$, the mean-field approximation can break down because the reacting particles cannot mix efficiently enough, but as the dimension of the system is increased above a certain "upper critical dimension" $d_{\mathrm{c}}$, such statistical anomalies disappear. For two diffusing reactants with a simple one-step reaction, it is known [5,8,21,44-47,51] that $d_{\mathrm{c}}=2 /(m+n-1)$. Since $d_{\mathrm{c}} \leq 2$ for $m, n \geq 1$, the mean-field approximation should be perfectly valid in the usual case $d=3$, which is consistent with experimental findings [2,36-43].

In contrast to the case of two diffusing reactants described above, relatively little is known $[4,8,14-16]$ about the case of one diffusing reactant $\left(D_{\mathrm{A}}>0\right)$ and one static reactant $\left(D_{\mathrm{B}}=0\right)$. This situation, depicted schematically in Fig. 1, describes the corrosion of a porous solid B saturated with a fluid solvent and exposed to an initially separated colloidal reactant A, as shown in recent electrochemical experiments (described below) [3]. Jiang and Ebner [4] first pointed out (for $m=n=1$ ) that setting $D_{\mathrm{B}}=0$ in (2b) is a non-trivial, i.e. singular, limit leading to different long-time behavior than in the case of $D_{\mathrm{B}}>0$ (no matter how small), which they explained with simple scaling arguments supported by Monte Carlo computer simulations. For an analytical description of such one-dimensional diffusion with one static reactant, we adopt the power-law form of the reaction term and study the coupled equations

$$
\begin{aligned}
& \frac{\partial \rho_{\mathrm{A}}}{\partial T}=D_{\mathrm{A}} \frac{\partial^{2} \rho_{\mathrm{A}}}{\partial X^{2}}-m^{\prime} k \rho_{\mathrm{A}}^{m} \rho_{\mathrm{B}}^{n}, \\
& \frac{\partial \rho_{\mathrm{B}}}{\partial T}=-n^{\prime} k \rho_{\mathrm{A}}^{m} \rho_{\mathrm{B}}^{n} .
\end{aligned}
$$

In the simplest case $m=n=1$, the initial-boundary-value problem (3)-(6a) and (6b) has been solved numerically by Havlin et al. [8] and analyzed in the limit of "long times" $T \rightarrow \infty$ by Koza [14], using various asymptotic approximations introduced by Gálfi and Rácz [1]. Rigorous analysis has been reported in the analogous limit of "fast reactions" $k \rightarrow \infty$ by Hilhorst et al. [15], but these authors only address the behavior at the diffusive length scale $X \propto \sqrt{T}$ (see Section 3.4) and do not consider the structure of the reaction front studied by Koza [14], which is of primary interest here. Hilhorst et al. [16] have also recently considered the effect of a more general reaction term at the diffusive scale, but the present work appears to be the first to analyze the nontrivial effect of changing reaction orders at the reactive length scale (see below) in the general case $(m, n \geq 1)$ with one static reactant.

The relevance of (6a) and (6b) for a given porous-solid corrosion system rests on several key assumptions that are less obviously satisfied a priori than in the case of two diffusing reactants. First, the solid matrix containing the static reactant $\mathrm{B}$ must be sufficiently porous that the moving reactant A can diffuse freely to the exposed surfaces with an effective diffusion constant (averaged over many pores) comparable to that in the bulk solvent. The concentration of A must also be dilute enough that $D_{\mathrm{A}}$ is constant. Another reason that the concentrations of A and B must be dilute is that the inert product $\mathrm{C}$ must be created in small enough quantities that its presence does not affect the reaction dynamics (e.g. by inhibiting diffusion or initiating convection). Finally, one might worry about the breakdown of the mean-field approximation since the (possibly fractal) pore structure may influence statistical averaging. For example, it is known that fluctuations alter the reaction-front dynamics when the diffusion is confined to a percolating cluster in two dimensions [8]. In spite of these concerns, however, the one-dimensional mean-field model (6a) and (6b) can in fact describe certain corrosion systems. 
An important motivation for the present analytical study is afforded by the recent experiments of Léger et al. [3], which are the first to examine in detail the case of one static and one diffusing reactant. These experiments involve the corrosion of ramified copper electrodeposits exposed to a cupric chloride electrolyte to produce cuprous chloride crystallites via the reaction

$$
\mathrm{CuCl}_{2}(\mathrm{aq})+\mathrm{Cu}(\text { solid }) \rightarrow 2 \mathrm{CuCl}(\text { solid })
$$

immediately following electrodeposition. It is found that the long-time behavior of (6a) and (6b) with $m=1$ matches the experimentally observed front speed and concentration profile of diffusing reactant $\left(\mathrm{CuCl}_{2}\right)$ rather well, in spite of the complex fractal geometry of the electrodeposits and the presence of the inert product $(\mathrm{CuCl})$ [3]. Since the reaction rate and the concentration of the static species $(\mathrm{Cu})$ are not directly measured, however, the interpretation of these kinds of corrosion experiments can be aided by the analysis presented here of the mean-field model with $m, n \geq 1$.

There is an extensive mathematical literature $[17,19,20,23,24]$ on the subject of single reaction-diffusion equations of the general form

$$
\frac{\partial \rho}{\partial T}=D \frac{\partial^{2} \rho}{\partial X^{2}}-f(\rho),
$$

which arise in many applications (e.g. chemical reactions, combustion and population dynamics). A common theme in these studies is the appearance of two distinct (time-dependent) length scales in the intermediate asymptotic regime $(t \rightarrow \infty)$ which correspond to either "weakly nonlinear behavior", where it has been established in many cases that the reaction term is negligible and the dynamics are purely diffusive, or "strongly nonlinear behavior", where the reaction and diffusion terms balance (in the nomenclature of Gmira and Veron [23]). This separation of scales also arises in coupled systems of reaction-diffusion equations like (2a) and (2b), but owing to their greater complexity, much less rigorous analysis has been reported. In the case of two diffusing reactants, Gálfi and Rácz [1] pointed out that if the diffusion constants are the same, $D_{\mathrm{A}}=D_{\mathrm{B}}$, then the difference in concentrations $\rho_{\mathrm{A}}-\rho_{\mathrm{B}}$ obeys a pure diffusion equation which can be easily integrated, thereby reducing the coupled system ( $2 a)$ and (2b) to a single equation with the form of (8). Another simplification occurs if also $\rho_{\mathrm{A}}^{0}=\rho_{\mathrm{B}}^{0}$, in which case the reaction front is perfectly symmetric and does not move. In this simplified case with $m=n=1$, Schenkel et al. [11] were able to prove that the asymptotic solution of Gálfi and Rácz [1], which combines different approximations at the diffusive and reactive scales, is approached uniformly as $T \rightarrow \infty$ starting from the initial conditions of (4), and they also reported rigorous bounds on the transient decay to the asymptotic solution. Recently, van Baalen et al. [12] have extended this analysis to the case of symmetric, high-order reactions $m=n>3$, where the reaction-front scaling is altered.

The analyses of Refs. [11,12] represent an important contribution because, at least in the case $D_{\mathrm{A}}=D_{\mathrm{B}}, \rho_{\mathrm{A}}^{0}=\rho_{\mathrm{B}}^{0}$ and $m=n$, they provide a rigorous mathematical justification for various ad hoc assumptions introduced by Gálfi and Rácz $[1,13,50]$ to describe the local structure of the reaction front which have otherwise been validated only by numerical simulations. Unfortunately, however, since the analysis in Refs. [11,12] relies on a comparison principle for single parabolic equations [22,23] of the form (8), it does not (as the authors indicate) appear to be applicable when $D_{\mathrm{A}} \neq D_{\mathrm{B}}$ (which also leads to a moving reaction front). Van Baalen et al. [12] also remark that their analysis is not easily extended to certain intermediate reaction orders $(1<m=n \leq 3)$. These difficulties are reflected in Koza's recent studies of the general cases $D_{\mathrm{A}}>D_{\mathrm{B}}>0[13,50]$ and $D_{\mathrm{B}}=0$ [14], in which several ad hoc (but reasonable) approximations are made and transients are ignored.

In the present article, the long-time asymptotics of the initial-boundary-value problem (3)-(6) are studied. This special case of $(2 a)$ and $(2 b)$ is more tractable analytically than the general case because (6b) can be integrated exactly in time, thereby reducing the coupled system to a single integro-partial differential equation. This useful 
simplification is presented in Section 2, where the problem is recast in a dimensionless form. It is also noted that similarity solutions are expected to exist because there is no natural length or time scale in the problem [24-26]. Although it may be possible to prove that the system actually approaches such a self-similar solution starting from the prescribed initial conditions, we instead pursue the more modest goal of proving that if an asymptotic similarity solution exists, it must have a certain unique form, i.e. we explore the consequences of the "quasi-stationary approximation" $[5,13,14,44-47,50]$. In Section 3 , the similarity solution is systematically derived, and it is shown that a "diffusion layer" (where the reaction term is dominated by the diffusion term) with different scaling properties than the "reaction front" (where the reaction and diffusion terms balance) must exist to satisfy the boundary conditions. In Section 4, the transient decay of the reaction rate in the diffusion layer is analyzed, thereby proving a posteriori that the reaction term can indeed be neglected in the dominant balance. In Section 5, a uniformly valid asymptotic approximation is constructed by matching the self-similar forms in the two different regions. Finally, in Section 6 some general physical conclusions are drawn from the analysis, and in Section 7 certain similarities are discussed between this work and the literature on combustion waves. (Note that Section 4 is more technical and may be skipped in a first reading.)

\section{Preliminaries}

\subsection{Dimensionless formulation}

With the definitions,

$$
\begin{aligned}
& t \equiv m^{\prime} k\left(\rho_{\mathrm{A}}^{0}\right)^{m-1}\left(\rho_{\mathrm{B}}^{0}\right)^{n} T, \quad x \equiv X \sqrt{m^{\prime} k\left(\rho_{\mathrm{A}}^{0}\right)^{m-1}\left(\rho_{\mathrm{B}}^{0}\right)^{n} / D_{\mathrm{A}}}, \\
& a(x, t) \equiv \rho_{\mathrm{A}}(X, T) / \rho_{\mathrm{A}}^{0}, \quad b(x, t) \equiv \rho_{\mathrm{B}}(X, T) / \rho_{\mathrm{B}}^{0},
\end{aligned}
$$

the initial-boundary-value problem (3)-(6) may be expressed in a dimensionless form

$$
\begin{aligned}
& \frac{\partial a}{\partial t}=\frac{\partial^{2} a}{\partial x^{2}}-a^{m} b^{n}, \\
& \frac{\partial b}{\partial t}=-q a^{m} b^{n}, \\
& a(\infty, t)=1, \quad b(\infty, t)=0, \quad a(-\infty, t)=0, \quad b(-\infty, t)=1, \\
& a(x, 0)=H(x), \quad b(x, 0)=H(-x),
\end{aligned}
$$

which involves only one dimensionless parameter

$$
q \equiv \frac{n^{\prime} \rho_{\mathrm{A}}^{0}}{m^{\prime} \rho_{\mathrm{B}}^{0}}
$$

Note that the dimensionless problem (10a)-(10d) depends only upon the initial concentrations $\rho_{\mathrm{A}}^{0}$ and $\rho_{\mathrm{B}}^{0}$ and the stochiometric coefficients $m^{\prime}$ and $n^{\prime}$ through the parameter $q$; the reaction rate $k$ and the diffusion constant $D_{\mathrm{A}}$ simply set the natural scales for length and time. From (9a), we see that the limit of "fast reactions" $k \rightarrow \infty$ (with $X$ and $T$ fixed) corresponds to the limit of long (dimensionless) times $t \rightarrow \infty$ at the diffusive scale $x \propto \sqrt{t}$. (See Ref. [15] for another discussion of this correspondence of limits.) 


\subsection{The governing integro-partial differential equation}

The statement of the problem (10a)-(10d) will be used in deriving the asymptotic similarity solution below, but for the transient analysis described in Section 4 it will be convenient to first integrate (10b) exactly in time. Note that (10b) and (10d) imply that $b(x, t)=0$ for $x>0$ at all times $t \geq 0$, which reflects the fact that species B cannot diffuse out of its initial region. For $x<0$, we integrate (10b) using the initial condition (10d) to express $b(x, t)$ as

$$
b(x, t)= \begin{cases}\mathrm{e}^{-q \phi_{m}(x, t)} & \text { if } n=1, x<0 \\ {\left[1+q(n-1) \phi_{m}(x, t)\right]^{-1 /(n-1)}} & \text { if } n \neq 1, x<0\end{cases}
$$

which involves the time-integral of $a(x, t)^{m}$ :

$$
\phi_{m}(x, t) \equiv \int_{0}^{t} a(x, \tau)^{m} \mathrm{~d} \tau .
$$

(Note that a partial differential equation satisfied by $\phi_{1}(x, t)$ is given in Ref. [15].) Substituting for $b(x, t)$ in $(10 a)$, we obtain a single, nonlinear integro-partial differential equation for $a(x, t)$, either

$$
\frac{\partial a(x, t)}{\partial t}=\frac{\partial^{2} a(x, t)}{\partial x^{2}}-H(x) a(x, t)^{m} \exp \left(-q \int_{0}^{t} a(x, \tau)^{m} \mathrm{~d} \tau\right)
$$

if $n=1$ or

$$
\frac{\partial a(x, t)}{\partial t}=\frac{\partial^{2} a(x, t)}{\partial x^{2}}-\frac{H(x) a(x, t)^{m}}{\left[1+q(n-1) \int_{0}^{t} a(x, \tau)^{m} \mathrm{~d} \tau\right]^{n /(n-1)}}
$$

if $n \neq 1$.

Although these equations involve only one unknown function $a(x, t)$, they are somewhat unwieldy, so we will first seek long-time $(t \rightarrow \infty)$ asymptotic solutions to the coupled system (10a)-(10d) in Section 3 . The time-dependent properties of (14) and (15) will be studied in Section 4. Before proceeding, however, we digress to show that physically meaningful solutions exist only if $n \geq 1$. Later in the analysis, we will see that $m \geq 1$ is required as well.

\subsection{A reaction front does not exist if $n<1$}

Consider any point $x_{0}<0$. Since species A diffuses to $x_{0}$ from a reservoir of constant concentration $(a(\infty, t)=1)$ while species B is removed by reactions without ever being replenished $\left(\partial b\left(x_{0}, t\right) / \partial t<0\right.$ for all $\left.t>0\right)$, it is clear that after long times $a\left(x_{0}, t\right)$ must eventually differ from zero. Therefore, there exists some $a_{*}\left(x_{0}\right)>0$ and $t_{0}>0$ such that $a\left(x_{0}, t\right)>a_{*}\left(x_{0}\right)$ for all $t>t_{0}$. This implies that $\phi_{m}\left(x_{0}, t\right)>a_{*}\left(x_{0}\right)^{m}\left(t-t_{0}\right)$ from (13) and thus $b\left(x_{0}, t\right) \rightarrow 0$ from (12) since $q>0$, but a singularity arises if $n<1$ : the concentration of static reactant $b\left(x_{0}, t\right)$ vanishes at some finite time $t_{1}$ given by $q(1-n) \phi_{m}\left(x_{0}, t_{1}\right)=1$ (which exists because $\phi\left(x_{0}, t\right)$ is continuous, $\phi_{m}\left(x_{0}, 0\right)=0$ and $\left.\phi_{m}\left(x_{0}, \infty\right)=\infty\right)$. For $t>t_{1}$, Eq. (12) predicts imaginary, negative, or diverging solutions for $n<1$, none of which are physically meaningful. Therefore, when $n<1$, the solutions to the model equations break down physically in a finite time, and in that sense there does not exist a stable, moving reaction front. Nevertheless, it should be noted that Hilhorst et al. $[15,16]$ have shown that well-defined solutions with free boundaries (at the diffusive scale $x \propto \sqrt{t}$ ) can exist when $0<n<1$. 


\section{Derivation of the asymptotic similarity solution}

\subsection{Scaling of the reaction front}

The initial-boundary-value problem (10a)-(10d) possesses no natural length or time scale, i.e. it is invariant under power-law "stretching transformations" [26], and consequently in the limit $t \rightarrow \infty$ the system is expected to approach an asymptotic similarity solution in which distance and time are coupled by power-law scalings [24,25]. Since reactant A diffuses while reactant B does not, the (presumably unique) point of maximal reaction rate $r(a, b)=a^{m} b^{n}$ moves in the $-x$ direction toward the reservoir of reactant $\mathrm{B}$. Therefore, an asymptotic similarity solution, if one exists, must involve a moving frame of reference centered on some point $x_{\mathrm{f}}(t)$ identifying the position of the reaction front at or near the point of maximal reaction rate (with $\mathrm{d} x_{\mathrm{f}} / \mathrm{d} t<0$ ). Let $x_{\mathrm{f}}(t)=-2 v t^{\sigma}$, where $v(q)>0$ is a constant (akin to the "speed" of the front) to be determined self-consistently during the analysis, and consider an arbitrary coordinate stretching transformation in the moving reference frame,

$$
\eta \equiv \frac{x+2 v t^{\sigma}}{t^{\alpha}}
$$

where $w(t)=t^{\alpha}$ is the width of the reaction front indicated in Fig. 1. (The factor of 2 is included only for algebraic convenience.)

In the neighborhood of $x_{\mathrm{f}}(t)$, we also allow the magnitude of $a(x, t)$ to vary with a power-law scaling,

$$
\tilde{\mathcal{A}}(\eta, t) \equiv t^{\gamma} a(x, t) .
$$

If $\gamma \neq 0$, then another similarity solution far away from the reaction front (in the "diffusion layer" shown in Fig. 1 and defined below) will be needed to satisfy the boundary condition $a(\infty, t)=1$. This possibility that $t$ wo regions with different asymptotically self-similar dynamics for $a(x, t)$ could arise is suggested by the fact that there are two driving terms, representing diffusion and reaction, on the right-hand side of (10a) with different behaviors under stretching transformations. On the other hand, there is only the reaction term on the right-hand side of (10b), so $b(x, t)$ can exhibit only one type of asymptotic scale invariance. This is the main mathematical consequence of the physical fact that reactant B does not diffuse. Since $b(-\infty, 1)=1$, we consider the transformation

$$
\tilde{\mathcal{B}}(\eta, t) \equiv b(x, t) .
$$

Note that the reaction term $r(a, b)=a^{m} b^{n}$ has the scaling $r=t^{-\beta} \tilde{\mathcal{A}}^{m} \tilde{\mathcal{B}}^{n}$, where $\beta=m \gamma$ in the notation of Gálfi and Rácz [1].

These transformations leave the governing equations in the form

$$
\begin{aligned}
& t^{(m-1) \gamma} \frac{\partial \tilde{\mathcal{A}}}{\partial t}-t^{(m-1) \gamma-1}\left(\gamma \tilde{\mathcal{A}}+\alpha \eta \frac{\partial \tilde{\mathcal{A}}}{\partial \eta}\right)+t^{(m-1) \gamma-1-\alpha+\sigma} 2 \sigma \nu \frac{\partial \tilde{\mathcal{A}}}{\partial \eta}=t^{(m-1) \gamma-2 \alpha} \frac{\partial^{2} \tilde{\mathcal{A}}}{\partial \eta^{2}}-\tilde{\mathcal{A}}^{m} \tilde{\mathcal{B}}^{n}, \\
& t^{m \gamma} \frac{\partial \tilde{\mathcal{B}}}{\partial t}-t^{m \gamma-1} \alpha \eta \frac{\partial \tilde{\mathcal{B}}}{\partial \eta}+t^{m \gamma-1-\alpha+\sigma} 2 \sigma \nu \frac{\partial \tilde{\mathcal{B}}}{\partial \eta}=-q \tilde{\mathcal{A}}^{m} \tilde{\mathcal{B}}^{n} .
\end{aligned}
$$

We now look for asymptotically invariant solutions

$$
\begin{aligned}
& \tilde{\mathcal{A}}(\eta, t) \rightarrow \mathcal{A}(\eta), \quad \frac{\partial \tilde{\mathcal{A}}}{\partial \eta}(\eta, t) \rightarrow \mathcal{A}^{\prime}(\eta), \quad \frac{\partial^{2} \tilde{\mathcal{A}}}{\partial \eta^{2}}(\eta, t) \rightarrow \mathcal{A}^{\prime \prime}(\eta), \\
& \tilde{\mathcal{B}}(\eta, t) \rightarrow \mathcal{B}(\eta), \quad \frac{\partial \tilde{\mathcal{B}}}{\partial \eta}(\eta, t) \rightarrow \mathcal{B}^{\prime}(\eta)
\end{aligned}
$$


as $t \rightarrow \infty$ with $|\eta|<\infty$ fixed. For consistency with our definition of the reaction front, we require that there is in each equation a dominant balance between the reaction term $\mathcal{A}^{m} \mathcal{B}^{n}$ and at least one other non-vanishing term. In order for time invariance to be attained, we assume that time-dependent terms in the transformed coordinates are negligible compared to the reaction term, i.e.

$$
\lim _{t \rightarrow \infty} t^{(m-1) \gamma} \frac{\partial \tilde{\mathcal{A}}}{\partial t}=\lim _{t \rightarrow \infty} t^{m \gamma} \frac{\partial \tilde{\mathcal{B}}}{\partial t}=0,
$$

which is a precise statement of the assumption of "quasi-stationarity" [14].

A dominant balance with the reaction term in (19a) implies that at least one of the following three cases must be true:

Case A1. $(m-1) \gamma-2 \alpha=0, \quad(m-1) \gamma-1-\alpha+\sigma \leq 0, \quad(m-1) \gamma-1 \leq 0$.

Case A2. $(m-1) \gamma-2 \alpha \leq 0, \quad(m-1) \gamma-1-\alpha+\sigma=0, \quad(m-1) \gamma-1 \leq 0$.

Case A3. $(m-1) \gamma-2 \alpha \leq 0, \quad(m-1) \gamma-1-\alpha+\sigma \leq 0, \quad(m-1) \gamma-1=0$.

Likewise a dominant balance in $(19 \mathrm{~b})$ requires that one of the following two cases must hold:

Case B1. $m \gamma-1=0, m \gamma-1-\alpha+\sigma \leq 0$.

Case B2. $m \gamma-1 \leq 0, m \gamma-1-\alpha+\sigma=0$.

There are only two combinations of these cases that are logically consistent:

Traveling wave case. (A2, B2) $\alpha \geq 0, \gamma=0, \sigma=1+\alpha$.

Diffusing front case. (A1, B2) $\alpha=\frac{1}{2}(m-1) \gamma, \sigma=1-\frac{1}{2}(m+1) \gamma, 0<\gamma \leq 1 / m$.

In the first case, we have $\sigma \geq 1$, which implies that the reaction front advances at least linearly, e.g. as a traveling wave $x_{\mathrm{f}} \sim t$, but in the second case, the front advances sublinearly, e.g. as a diffusing front $x_{\mathrm{f}} \sim t^{1 / 2}$. In both cases, note that the reaction order $n$ of the static species B plays no role in the scaling behavior. The same conclusion is also true of the reaction order $m$ of the diffusing species A in the traveling wave case, but $m$ does affect the scaling exponents in the diffusing front case.

Consider the possibility $\gamma=0$, which is only consistent with the traveling wave case. In this case, a single asymptotic scale invariance is attained everywhere, and the equations for $\mathcal{A}(\eta)$ and $\mathcal{B}(\eta)$ are

$$
\begin{aligned}
& 2 \sigma \nu \mathcal{A}^{\prime}=\delta_{\alpha, 0} \mathcal{A}^{\prime \prime}-\mathcal{A}^{m} \mathcal{B}^{n}, \\
& 2 \sigma \nu \mathcal{B}^{\prime}=-q \mathcal{A}^{m} \mathcal{B}^{n},
\end{aligned}
$$

where $\delta_{x, y}$ is the Kronecker delta. By combining these equations and integrating once using the boundary conditions behind the front, i.e. $\mathcal{A}(\infty)=1, \mathcal{A}^{\prime}(\infty)=0$ and $\mathcal{B}(\infty)=0$, we obtain

$$
2 \sigma v(\mathcal{B}+q)=q\left(2 \sigma \nu \mathcal{A}-\delta_{\alpha, 0} \mathcal{A}^{\prime}\right) .
$$

Applying the boundary conditions ahead of the front, $\mathcal{A}(-\infty)=\mathcal{A}^{\prime}(-\infty)=0$ and $\mathcal{B}(-\infty)=1$, this equation then implies that $\sigma v(1+q)=0$, which is a contradiction since $\sigma>0$ and $v>0$ are needed for the reaction front to move at all (and $q>0$ ).

In this way, we are forced to consider at least two regions with different scale invariance if there is to be any hope of an asymptotic similarity solution. Since the second type of scale invariance is associated with the dominance of the diffusion term versus the reaction term in (10a), it must occur only on the back $(+x)$ side of the reaction front due to the reservoir of reactant A at infinity, $a(\infty, t)=1$ (see Fig. 1). To describe the scale invariance of the diffusion layer, we postulate another power law $W(t)=t^{\delta}$ for the asymptotic width of the diffusion layer.

\subsection{Scaling of the diffusion layer}

Since $\delta \neq \alpha$, there are two possibilities, each involving a singular perturbation $w / W=t^{\alpha-\delta}$ : 
Infinitely thin reaction front case. $\delta>\alpha, w=\mathrm{o}(W)$.

Infinitely thin diffusion layer case. $\delta<\alpha, W=\mathrm{o}(w)$.

Since chemical reactions are typically much faster than diffusion, the former case seems more reasonable on physical grounds, but we do not rule out the latter case a priori. In the infinitely thin reaction front case, the reaction front is defined by $x-x_{\mathrm{f}}=\mathrm{O}(w)$ and the diffusion layer by $W=\mathrm{O}\left(x-x_{\mathrm{f}}\right), x>x_{\mathrm{f}}$, whereas in the infinitely thin diffusion layer case, the reaction front is defined by $w=\mathrm{O}\left(x-x_{\mathrm{f}}\right), x<x_{\mathrm{f}}$ and the diffusion layer by $x-x_{\mathrm{f}}=\mathrm{O}(W)$. In both cases, we view the reaction front as representing the "inner problem" (with similarity variable $|\eta|<\infty$ ) and the diffusion layer as representing the "outer problem" (with similarity variable $\zeta>0$ defined below). The two regions are connected by asymptotic matching of the limits $\eta \rightarrow \infty$ and $\zeta \rightarrow 0^{+}$(described in Section 3.3) [27,28].

In order to treat the outer problem, we transform the original equations using a new reduced coordinate with power-law scalings,

$$
\zeta \equiv \frac{x+2 v t^{\sigma}}{2 t^{\delta}}, \quad \tilde{A}(\zeta, t) \equiv a(x, t), \quad \tilde{B}(\zeta, t) \equiv b(x, t)
$$

(Another factor of 2 is included in $\eta=2 t^{\delta-\alpha} \zeta$ for algebraic convenience. Note the use of A and B for the diffusion layer versus $\mathcal{A}$ and $\mathcal{B}$ for the reaction front.) Under this transformation, the equations take the form

$$
\begin{aligned}
& t^{2 \delta} \frac{\partial \tilde{A}}{\partial t}-t^{2 \delta-1} \delta \zeta \frac{\partial \tilde{A}}{\partial \zeta}+t^{\delta+\sigma-1} \sigma \nu \frac{\partial \tilde{A}}{\partial \zeta}=\frac{1}{4} \frac{\partial^{2} \tilde{A}}{\partial \zeta^{2}}-t^{2 \delta} \tilde{A}^{m} \tilde{B}^{n}, \\
& t^{2 \delta} \frac{\partial \tilde{B}}{\partial t}-t^{2 \delta-1} \delta \zeta \frac{\partial \tilde{B}}{\partial \zeta}+t^{\delta+\sigma-1} \sigma \nu \frac{\partial \tilde{B}}{\partial \zeta}=-t^{2 \delta} q \tilde{A}^{m} \tilde{B}^{n} .
\end{aligned}
$$

Seeking an asymptotic similarity solution, we assume that invariance is achieved in the transformed equations

$$
\begin{array}{rlrl}
\tilde{A}(\zeta, t) & \rightarrow A(\zeta), & \frac{\partial \tilde{A}}{\partial \zeta}(\zeta, t) \rightarrow A^{\prime}(\zeta), & \frac{\partial^{2} \tilde{A}}{\partial \zeta^{2}}(\zeta, t) \rightarrow A^{\prime \prime}(\zeta), \\
\tilde{B}(\zeta, t) \rightarrow B(\zeta), & \frac{\partial \tilde{B}}{\partial \zeta}(\zeta, t) \rightarrow B^{\prime}(\zeta), &
\end{array}
$$

assuming time-variations in (26a) are small relative to the diffusion term

$$
\lim _{t \rightarrow \infty} t^{2 \delta} \frac{\partial \tilde{A}}{\partial t}(\zeta, t)=0 \quad \text { for } \zeta>0 .
$$

In order to obtain a different scaling from the reaction front, the reaction term must also not enter into the dominant balance

$$
\lim _{t \rightarrow \infty} t^{2 \delta} \tilde{A}(\zeta, t)^{m} \tilde{B}(\zeta, t)^{n}=0 \quad \text { for } \zeta>0
$$

a condition that we will check a posteriori for consistency in Section 4. Note that this limit vanishes trivially for $\zeta>v$ since we have already noted that $b(x, t)=0$ for all $x>0$. From (25b), this condition would imply $\partial \tilde{B} / \partial \zeta=0$, which together with the boundary condition $\tilde{B}(\infty)=0$ would imply $\tilde{B}(\zeta)=0$. With the reaction term gone, one of the terms on the left-hand side of (25a) must balance the $\partial^{2} \tilde{A} / \partial \zeta^{2}$ term on the right-hand side; if not, we would have $\partial^{2} \tilde{A} / \partial \zeta^{2}=0$, which cannot satisfy all of the boundary conditions. There are only two possible dominant balances:

Case D1. $\delta+\sigma-1=0$ and $2 \delta-1 \leq 0$.

Case D2. $\delta+\sigma-1 \leq 0$ and $2 \delta-1=0$. 
The former case implies $\sigma>\frac{1}{2}$ and hence contains the traveling wave case (and not the diffusing front case). With the scaling relations of case D1, Eq. (25a) has the asymptotic form

$$
\sigma v A^{\prime}=\frac{1}{4} A^{\prime \prime}
$$

The solutions to this equation exhibit exponential growth as $\zeta \rightarrow \infty$, which is incompatible with the boundary condition $A(\infty)=1$. Therefore, we conclude that case $\mathrm{D} 1$, and hence also the traveling wave case, is not consistent with the boundary conditions. At this point, we are left with case D2 together with the diffusing front case (A1, B2), which imply $W \sim \sqrt{t}$, thus justifying the term "diffusion layer" for the region $\zeta>0$.

\subsection{Asymptotic matching of the reaction front and diffusion layer}

One more condition is needed to uniquely determine the scaling exponents, and it comes from asymptotic matching. The "outer limit" $\eta \rightarrow \infty$ of the inner approximation must match with the "inner limit" $\zeta \rightarrow 0^{+}$ of the outer approximation (because both are asymptotic representations of the same function). Unlike the more familiar case of boundary layers of ordinary differential equations [27,28], however, our system of partial differential equations will require extra care for matching because the limit $t \rightarrow \infty$ (with either $\zeta$ or $\eta$ fixed) must be taken before the inner and outer limits.

Since $B(\zeta)=0$ for all $\zeta>0$, the only matching condition for $b(x, t)$ is trivial, $\mathcal{B}(\infty)=0$, but the matching conditions for $a(x, t)$ are more subtle. Since $\gamma>0$, the concentration of species A approaches 0 in the reaction front: $a(x, t)=\mathrm{O}\left(t^{-\gamma}\right)$ as $t \rightarrow \infty$ with $|\eta|<\infty$ fixed. Therefore, a boundary condition on the outer problem is $A(\zeta)=0$, but unfortunately this does not provide a boundary condition on the inner problem. Instead, we must consider matching at the next (linear) order of Taylor expansion in the intermediate region:

$$
\frac{\partial a}{\partial x}= \begin{cases}\frac{\partial \tilde{A}}{\partial \zeta} \frac{\partial \zeta}{\partial x} \sim \frac{A^{\prime}(\zeta)}{2 t^{\delta}} & \text { as } t \rightarrow \infty \text { with } 0<\zeta<\infty \text { fixed } \\ \frac{1}{t^{\gamma}} \frac{\partial \tilde{\mathcal{A}}}{\partial \eta} \frac{\partial \eta}{\partial x} \sim \frac{\mathcal{A}^{\prime}(\eta)}{t^{\alpha+\gamma}} & \text { as } t \rightarrow \infty \text { with }|\eta|<\infty \text { fixed. }\end{cases}
$$

Now requiring that the two intermediate limits match yields the final scaling relation

$$
\alpha+\gamma=\delta
$$

as well as the missing boundary condition on the inner problem

$$
\mathcal{A}^{\prime}(\infty)=\frac{1}{2} A^{\prime}(0)
$$

(Note that $A(\zeta)$ is already fully determined by matching at zeroth order.) This scaling relation (31) can be understood physically as expressing conservation of mass between the diffusion layer and reaction front [4]. Similarly, the matching condition (32) simply means that the diffusive flux entering the reaction front equals the flux leaving the diffusion layer.

By examining all possible similarity solutions with power-law couplings of distance and time, we finally arrive at a unique set of scaling exponents from cases A1, B2 and D2 and (31):

$$
\alpha=\frac{m-1}{2(m+1)}, \quad \beta=\frac{m}{m+1}, \quad \gamma=\frac{1}{m+1}, \quad \sigma=\delta=\frac{1}{2} .
$$

Therefore, after long times the reaction front itself "diffuses" according to $x_{\mathrm{f}}(t)=-2 v \sqrt{t}$, where $v(q)^{2}$ is now interpreted as an effective diffusion constant for the front. Although for $m=1$ the reaction zone settles down 
to a constant width $(\alpha=0)$, for $m>1$ the front width grows in time $(\alpha>0)$. In all cases the reaction front is "infinitely thin" compared to the diffusion layer $(\alpha<\delta)$. Note that as $m$ increases, $\gamma$ tends to zero, meaning that the concentration in the reaction front does not decrease as quickly for higher-order reactions as it does for first-order reactions. The exponents $\alpha=0$ and $\gamma=\frac{1}{2}$ for the case $m=1$ were first obtained by Jiang and Ebner [4] based on physical arguments supported by Monte Carlo simulations and later discussed in an analytical context by Koza [14], but to our knowledge prior to this work neither have the general expressions for $m \neq 1$ been given nor have the scaling exponents been proven to be unique. With the scaling exponents and matching boundary conditions now determined, we proceed to solve the inner and outer boundary-value problems in the following sections.

\subsection{Concentration profiles in the diffusion layer}

In the diffusion layer from (25)-(28), we have

$$
-2(\zeta-v) A^{\prime}=A^{\prime \prime}, \quad A(0)=0, \quad A(\infty)=1
$$

The exact solution to this boundary value problem can be expressed in terms of error functions [30]:

$$
A(\zeta)=\frac{\operatorname{erf}(\zeta-v)+\operatorname{erf}(v)}{1+\operatorname{erf}(v)}
$$

and is depicted in Fig. 2. Note that the dimensionless flux entering the reaction front

$$
\mathcal{A}_{1}(v) \equiv \frac{A^{\prime}(0)}{2}=\frac{\mathrm{e}^{-v^{2}}}{\sqrt{\pi}(1+\operatorname{erf}(v))}
$$

is needed for asymptotic matching in (32).

The effect of varying $q=n^{\prime} \rho_{\mathrm{A}}^{0} / m^{\prime} \rho_{\mathrm{B}}^{0}$ is easily understood in terms of the mathematical model. As $q$ is decreased, the reaction front slows down since reactions in the front region remove species A much faster than diffusion can replenish it. In the limit $q \rightarrow 0$, i.e. $\rho_{\mathrm{A}}^{0} \rightarrow 0$, the front comes to a complete stop, $v(0)=0$. For very small, but finite $q>0$, the concentration of diffusing reactant approximately obeys

$$
\frac{\partial a}{\partial t}=\frac{\partial^{2} a}{\partial x^{2}}, a(x, 0)=H(x), a(0, t)=0, a(\infty, t)=1, x \geq 0
$$

(at least for $t \ll v^{-2}$ since the reaction front is stationary only for short times). This classical diffusion problem has the exact similarity solution

$$
a(x, t)=\operatorname{erf}\left(\frac{x}{2 \sqrt{t}}\right),
$$

which is precisely the $v=0$ curve in Fig. 2. From (35), note that even when the front has moved significantly $\left(t \gg v^{-2}\right)$, the concentration still has the same shape, $A(\zeta) \approx \operatorname{erf}(\zeta)$, in the (very slowly) moving reference frame as long as $v(q) \ll 1$.

On the other hand, as $q$ is increased, the reaction term becomes progressively less important compared to the diffusion term in (10a). In the limit $q \rightarrow \infty$, i.e. $\rho_{\mathrm{B}}^{0} \rightarrow 0$, we recover another classical diffusion problem (after sufficiently long times $t \gg v^{-2}$ )

$$
\frac{\partial a}{\partial t}=\frac{\partial^{2} a}{\partial x^{2}}, a(x, 0)=H(x), a(-\infty, t)=0, a(\infty, t)=1,
$$



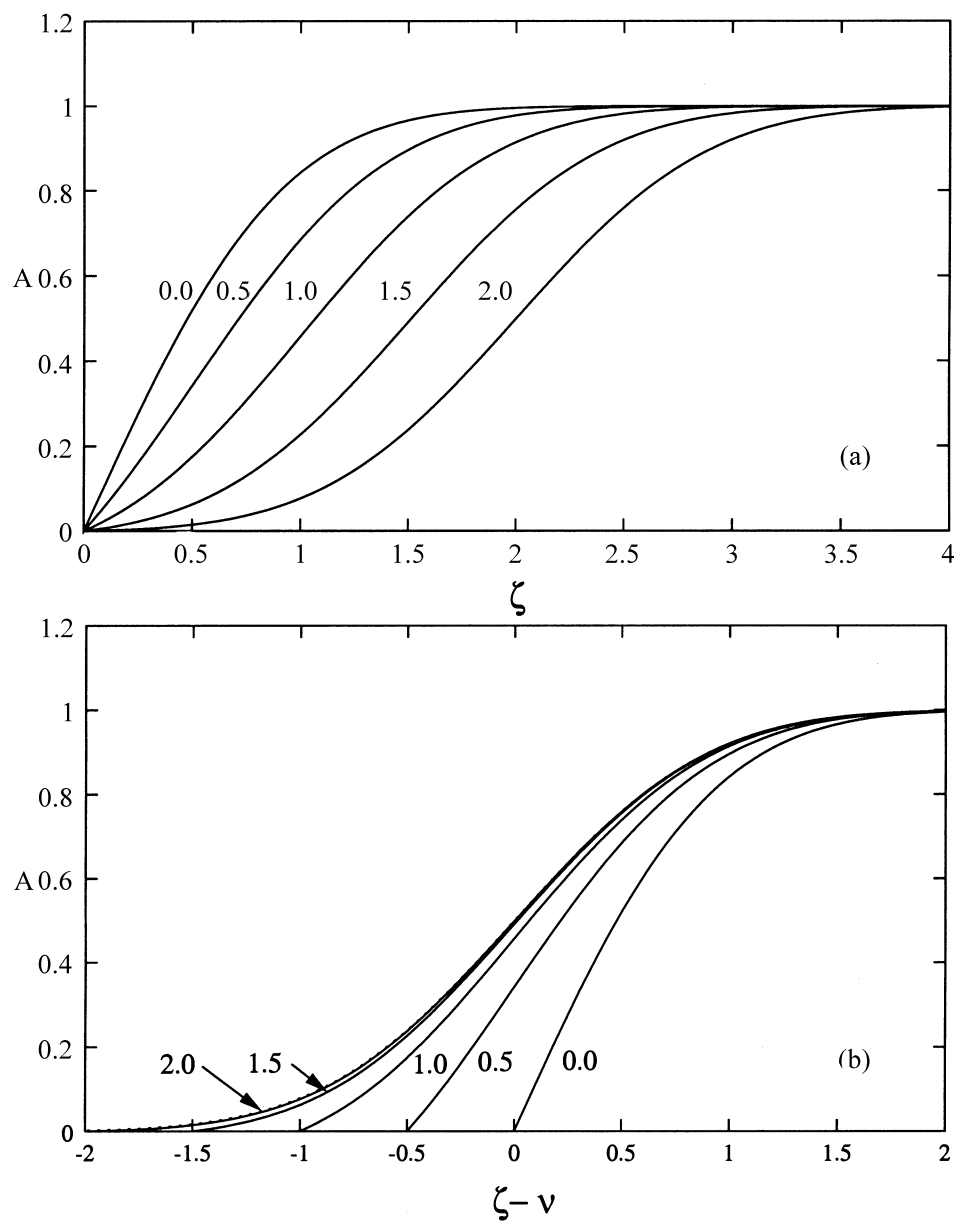

Fig. 2. The asymptotic similarity function in the diffusion layer, $a(x, t) \sim A(\zeta)$, where $\zeta=v+x / 2 \sqrt{t}$ is shown for $v=0.0,0.5,1.0,1.5,2.0$ versus $\zeta$ in (a) and versus $\zeta-v$ in (b). The limiting shape $\operatorname{erf}(x / 2 \sqrt{t})$ corresponds to $v=0$. The other limiting shape $\frac{1}{2}[1+\operatorname{erf}(x / 2 \sqrt{t})]$ as $v \rightarrow \infty$ is plotted as the dashed line in (b), but it is almost indistinguishable from the $v=2$ curve.

which has the exact similarity solution

$$
a(x, t)=\frac{1}{2}\left[1+\operatorname{erf}\left(\frac{x}{2 \sqrt{t}}\right)\right]
$$

Note that in the limit $q \rightarrow \infty$, the reaction front instantly speeds off to $-\infty$ having consumed only a negligible amount of reactant $\mathrm{A}$, resulting in a pure diffusion problem for $a(x, t)$. Indeed, we will see below that $v(\infty)=\infty$. It remains, of course, to relate $v$ and $q$.

In Fig. 2(b), we see how the true asymptotic similarity solution $A(\zeta)$ interpolates between the limiting forms (38) and (40) as $v$ goes from 0 to $\infty$, respectively. It turns out that for $v \geq 2$, the asymptotic behavior of the original reaction-diffusion system is almost indistinguishable from (40) for $t \gg \frac{1}{4}$. 


\subsection{Diffusion constant of the reaction front}

In the reaction front, we have a third-order system of nonlinear ordinary differential equations

$$
\begin{aligned}
& 0=\mathcal{A}^{\prime \prime}-\mathcal{A}^{m} \mathcal{B}^{n}, \\
& \nu \mathcal{B}^{\prime}=-q \mathcal{A}^{m} \mathcal{B}^{n},
\end{aligned}
$$

with four boundary conditions

$$
\mathcal{A}(-\infty)=0, \quad \mathcal{B}(-\infty)=1, \quad \mathcal{B}(\infty)=0 \quad \text { and } \quad \mathcal{A}^{\prime}(\infty)=\mathcal{A}_{1},
$$

where $\mathcal{A}_{1}(v)$ is known via (36). Although this boundary-value problem appears to be overdetermined, the fourth boundary condition is actually necessary to determine the unknown diffusion constant of the reaction front $v(q)$.

By comparing (19a) and (19b) and (41a) and (41b), some physical insight into the dynamics of the reaction front is gained. The concentration of diffusing reactant A is determined by a local balance of reactions and "steady state" diffusion and the concentration of static reactant B by a local balance of reactions and fictitious advection due to the translating reference frame. The latter balance reflects the special character of $D_{\mathrm{B}}=0$ : since reactant B cannot diffuse to the front, instead the front must diffuse to it. This is no longer true if $D_{\mathrm{B}}>0$ (no matter how small), which explains why different scaling exponents arise in that case [1,4]. These physical properties are manifested in the mathematical model by the fact that since it multiplies the highest derivative in the equations, $D_{\mathrm{B}}>0$ is a singular perturbation.

One integration of (41a) and (41b) is easy to perform and fortunately suffices to derive an exact expression for $v(q)$. Substituting (41b) into (41a), integrating and applying the boundary conditions at $\eta=\infty$, we obtain

$$
\nu \mathcal{B}=q\left(\mathcal{A}_{1}-\mathcal{A}^{\prime}\right) .
$$

Likewise enforcing the boundary conditions at $\eta=-\infty$, we find $v=q \mathcal{A}_{1}$. Substituting $\mathcal{A}_{1}$ from (36), we have

$$
v(q)=F^{-1}(q),
$$

where

$$
F(x) \equiv \sqrt{\pi} x \mathrm{e}^{x^{2}}[1+\operatorname{erf}(x)]
$$

which was first derived by Koza [14]. The function $v(q)$ is plotted in Fig. 3.

The transcendental function $F(x)$ cannot be inverted analytically, but limiting formulae can be derived. The Maclaurin series of $F(x)$ is

$$
F(x)=\sqrt{\pi} x+2 x^{2}+\sqrt{\pi} x^{3}+\frac{4}{3} x^{4}+\frac{\sqrt{\pi}}{2} x^{5}+\frac{8}{15} x^{6}+\frac{\sqrt{\pi}}{6} x^{7}+\cdots,
$$

which can be inverted term by term to generate the Maclaurin series of $v(q)$, valid for small $q$,

$$
v(q)=\frac{1}{\pi^{1 / 2}} q-\frac{2}{\pi^{3 / 2}} q^{2}+\frac{8-\pi}{\pi^{5 / 2}} q^{3}-\cdots .
$$

For large $q$, approximations such as

$$
v(q) \sim \sqrt{\log \left(\frac{q}{\sqrt{\pi}}\right)-\log 2-\frac{1}{2} \log \log \left(\frac{q}{\sqrt{\pi}}\right)}
$$

can be generated by iteration. 


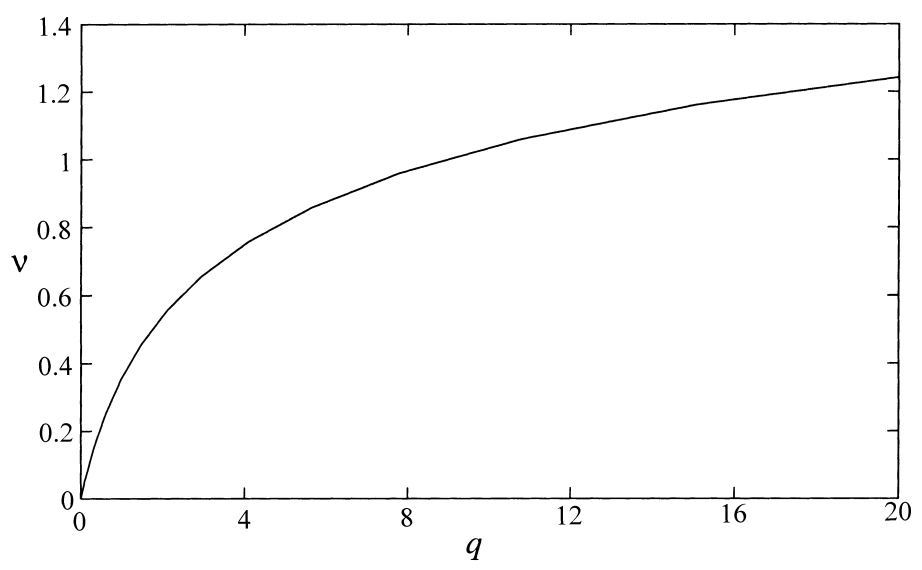

Fig. 3. The exact dependence of $v$, (the square root of) the dimensionless diffusion constant of the reaction front, on the parameter $q=n^{\prime} \rho_{\mathrm{A}}^{0} / m^{\prime} \rho_{\mathrm{B}}^{0}$ from Eq. (44).

\subsection{Existence and uniqueness of the reaction-front scaling functions}

With the results of the previous section, the inner boundary-value problem is reduced to a nonlinear second-order equation for $\mathcal{A}(\eta)$ :

$$
\mathcal{A}^{\prime \prime}=\mathcal{A}^{m}\left(1-\frac{\mathcal{A}^{\prime}}{\mathcal{A}_{1}}\right)^{n}, \quad \mathcal{A}(-\infty)=0, \quad \mathcal{A}^{\prime}(\infty)=\mathcal{A}_{1}
$$

Once this system is solved, $\mathcal{B}(\eta)$ is recovered from $\mathcal{B}(\eta)=1-\mathcal{A}^{\prime}(\eta) / \mathcal{A}_{1}$. Note that (49) is invariant under translation $\eta \mapsto \eta-\eta_{0}$, where the arbitrary constant $\eta_{0}$ sets the precise location of the reaction front. Since $\eta_{0}$ depends on the exact initial conditions, however, it cannot be determined by considering only the long-time asymptotic limit as we have done here.

Since the second-order equation (49) is autonomous (i.e. $\eta$ does not appear), it is useful to consider the "Lie diagram" [26] or "phase plane" [28,29] of trajectories in the $(\mathcal{A}, \mathcal{B})$ plane parameterized by $\eta$, as shown in Fig. 4. By studying properties of the phase plane, it is straightforward to prove the existence and uniqueness of solutions if and only if $m, n \geq 1$ and $\mathcal{A}_{1}>0$. With the change of variables,

$$
s \equiv \mathcal{A}_{1}^{(m-1) /(m+1)} \eta, \quad u(s) \equiv \mathcal{B}(\eta), \quad v(s) \equiv \mathcal{A}_{1}^{-2 /(m+1)} \mathcal{A}(\eta),
$$

we begin by transforming (49) into a system of first-order equations

$$
\begin{aligned}
& u^{\prime}=-v^{m} u^{n}, \\
& v^{\prime}=1-u,
\end{aligned}
$$

with boundary conditions $v(-\infty)=0$ and $u(\infty)=0$, or equivalently $u(-\infty)=1$ and $v^{\prime}(\infty)=1$.

There is a unique fixed point at $(u, v)=(1,0)$ corresponding to the region ahead of the reaction front which contains only the static reactant $\mathrm{B}$. This is the starting point $(s=-\infty)$ of any trajectories that satisfy the boundary condition $v(-\infty)=0$, so our task is to identify and follow any unstable manifolds leaving $(1,0)$ to see if they satisfy the other boundary condition $u(\infty)=0$. If $m=1$, then the equations can be linearized about the fixed point, and $(1,0)$ is a hyperbolic saddle point with an unstable manifold in the $(1,-1)$ direction and a stable manifold in the $(1,1)$ direction, as shown in Fig. 4(a). 

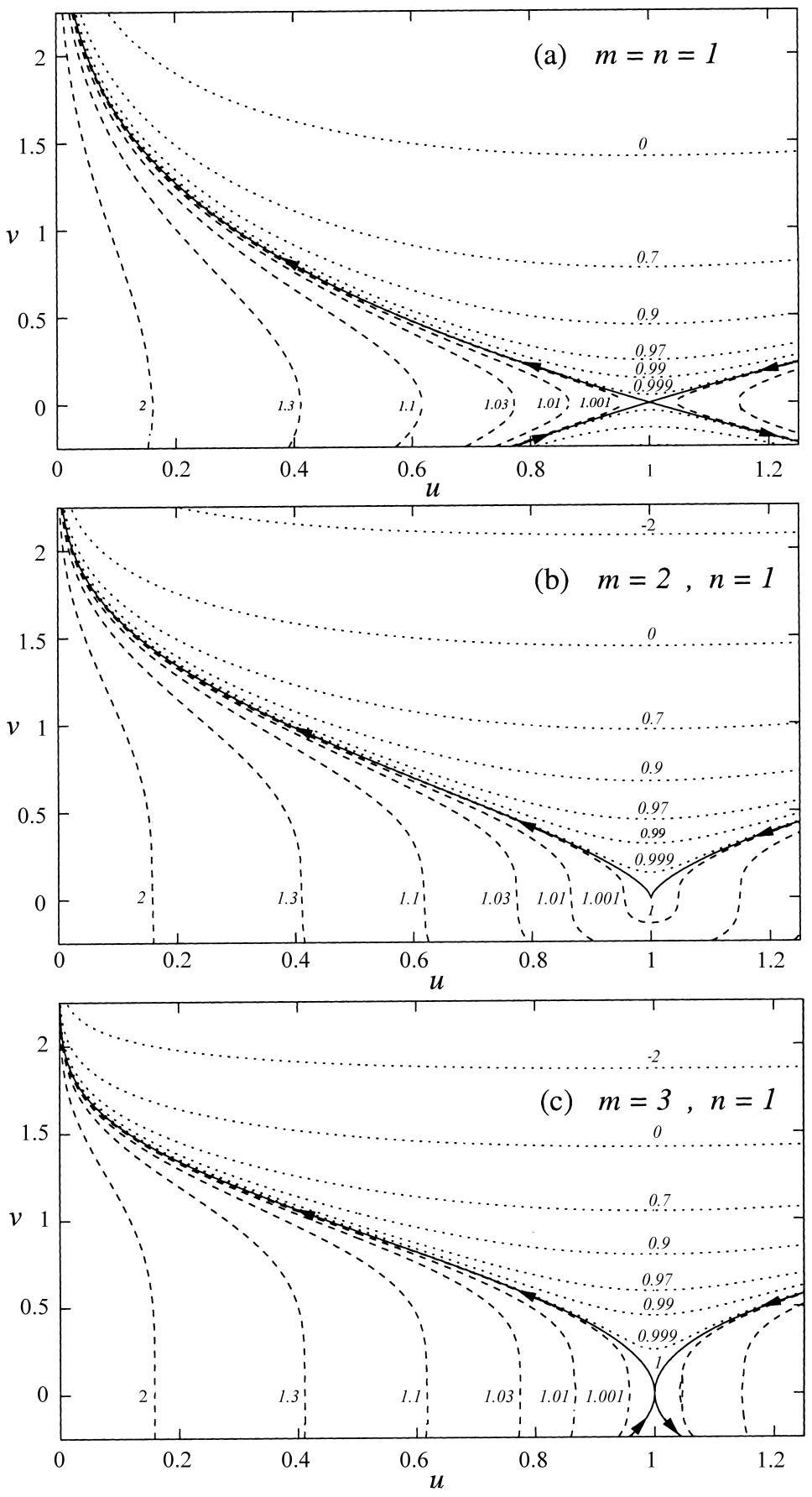

Fig. 4. Phase-plane trajectories for the inner (reaction-front) boundary-value problem from Eq. (55) labeled by the constant $c_{n}$ for $m=n=1$ in (a), $m=2, n=1$ in (b) and $m=3, n=1$ in (c). In each case, the solid lines are separatrices emanating from the fixed point $(1,0)$ of which the solution to the inner problem corresponds to the unique curve connecting $(1,0)$ and $(0, \infty)$. Arrows indicate the direction of increasing $s$. 
If $m \neq 1$, then the stable and unstable manifolds are degenerate at linear order and form a cusp oriented in the $(0,1)$ direction, as shown in Figs. 4(b) and (c). The nonlinear stability of the fixed point can be determined by noting that $v^{\prime \prime} \sim v^{m}$ as $u \rightarrow 1$. This equation has solutions satisfying the boundary condition $v(-\infty)=0$ if and only if $m \geq 1$. A stable reaction front does not exist if $m<1$ because the concentration of diffusing reactant A would become negative ahead of the front. Therefore, since $m \geq 1$ implies $\alpha=(m-1) / 2(m+1) \geq 0$, the front width $w(t) \sim t^{\alpha}$ either stays the same (for $m=1$ ) or increases (for $m>1$ ) but cannot decrease in time. For $m \geq 1$, we integrate $v^{\prime \prime} \sim v^{m}$ once and substitute into (51b) to obtain the separatrices in the upper half-plane $(v>0)$ :

$$
u \sim 1 \pm \sqrt{\frac{2}{m+1}} v^{(m+1) / 2} \quad \text { as }(u, v) \rightarrow\left(1,0^{+}\right)
$$

where the upper sign corresponds to the stable manifold $(\eta \rightarrow \infty)$ and the lower sign to the unstable manifold $(\eta \rightarrow-\infty)$.

Let us briefly consider trajectories in the lower half-plane $(v<0)$ in the neighborhood of the fixed point $(u \approx 1)$. Of course, such trajectories are not physically allowed, but it is satisfying to prove that the model equations exclude such possibilities. If $m$ is either an irrational or a rational number of the irreducible form $k_{1} / k_{2}$ where $k_{2}$ is even, then such trajectories do not exist because in that case $v^{m}$ (and hence $u^{\prime}$ ) would not be a real number. If $m=k_{1} / k_{2}$ where $k_{1}$ is even and $k_{2}$ is odd, then the direction field $\left(u^{\prime}, v^{\prime}\right)$ is an even function of $v$, which in light of (52) implies that there are no other separatrices in the lower half-plane and that trajectories merely circle the fixed point, as shown in Fig. 4(b). Finally, if $m=k_{1} / k_{2}$ where both $k_{1}$ and $k_{2}$ are odd, then $u^{\prime}$ is an odd function of $v$ (while $v^{\prime}$ is even), and $(1,0)$ is a saddle point. In this case, Eq. (52) also describes separatrices in the lower half-plane with the lower sign corresponding to the stable manifold and the upper to the unstable manifold, as shown in Fig. 4(c). As it leaves the fixed point, this branch of the unstable manifold enters the region $(u>1, v<0)$, throughout which $u^{\prime}>0$ and $v^{\prime}<0$, and thus it heads off to $u=v=-\infty$ and cannot satisfy the other boundary condition, as shown in Figs. 4(a) and (c). Therefore, any solutions must lie entirely in the first quadrant of the phase plane $(u>0, v>0)$.

In this way we are left with only one possible solution, which leaves the fixed point along the unstable manifold of (52) and enters the region defined by $0<u<1$ and $v>0$, throughout which $u^{\prime}<0$ and $v^{\prime}>0$. Since the $v$-axis $(u=0)$ is itself a trajectory, which cannot be crossed, this candidate solution must reach an asymptote $u(\infty)=u_{0}$ for some constant $0 \leq u_{0}<1$. However, it is clear from (51a) that $u_{0}=0$ is the only possible asymptote, which implies that the trajectory (if it exists) must satisfy the other boundary condition $u(\infty)=0$. To check the existence of this solution in the limit $s \rightarrow \infty$, note that $v^{\prime} \sim 1$ which implies $u^{\prime} \sim-\left(s-s_{0}\right)^{m} u^{n}$ for some constant $s_{0}$ with $m>1$. Solutions to this equation satisfying $u(\infty)=0$ exist if and only if $n \geq 1$. A stable reaction front does not exist if $n<1$ because the concentration of static reactant B would be negative behind the front.

\subsection{Concentration profiles in the reaction front}

Although solutions to (49) exist for $m, n \geq 1$, they are not easily expressed in terms of elementary functions. The exact trajectories in the phase plane, however, can be obtained. The ratio of (51b) and (51a) yields a separable first-order equation for $v(u)$ :

$$
\frac{\mathrm{d} v}{\mathrm{~d} u}=\frac{u-1}{v^{m} u^{n}}
$$


which can be integrated to obtain the one-parameter family of trajectories (for $u>0$ ) plotted in Fig. 4:

$$
(m+1)^{-1} v^{m+1}= \begin{cases}c_{1}+u-\log u & \text { if } n=1, \\ c_{2}+\log u+u^{-1} & \text { if } n=2, \\ c_{n}-(n-2) u^{2-n}+(n-1)^{-1} u^{1-n} & \text { if } n>1, n \neq 2,\end{cases}
$$

indexed by the real number $c_{n}$.

Applying the boundary conditions $v(u=1)=0$ (which determines $c_{n}$ ) and $v(0)=\infty$ (which selects the positive branch when $v(u)$ is multivalued), we arrive at the exact phase-plane trajectories (in the region $0<u<1, v>0$ ) of the solution to the inner problem:

$$
v= \begin{cases}{[(m+1)(u-1-\log u)]^{1 /(m+1)}} & \text { if } n=1, \\ {\left[(m+1)\left(u^{-1}-1+\log u\right)\right]^{1 /(m+1)}} & \text { if } n=2, \\ {\left[\frac{(m+1)\left(1-(n-1) u^{2-n}+(n-2) u^{1-n}\right)}{(n-1)(n-2)}\right]^{1 /(m+1)}} & \text { if } n>1, n \neq 2,\end{cases}
$$

which is an algebraic equation $v=g_{m, n}(u)$ relating $\mathcal{A}(\eta)$ and $\mathcal{B}(\eta)$ via (50). This equation is transcendental, but in some cases it is easily solved for $u=g_{m, n}^{-1}(v)$, e.g. for $n=3$ we have

$$
u=g_{m, 3}^{-1}(v)=\frac{\sqrt{2(m+1)^{-1} v^{m+1}}-1}{2(m+1)^{-1} v^{m+1}-1} .
$$

Note that $g_{m, n}^{-1}(0)=1$ and $g_{m, n}^{-1}(\infty)=0$.

By substituting (55) into (51b), we arrive at a first-order equation for $v(\eta)$ :

$$
v^{\prime}=1-g_{m, n}^{-1}(v)
$$

without any boundary conditions (because the conditions $v(-\infty)=0$ and $v^{\prime}(\infty)=1$ are automatically satisfied). Since (57) is separable, the solution to the inner problem can be expressed in the form

$$
\begin{aligned}
& \mathcal{A}(\eta)=\mathcal{A}_{1}^{2 /(m+1)} h_{m, n}^{-1}\left(\mathcal{A}_{1}^{(m-1) /(m+1)}\left(\eta-\eta_{0}\right)\right), \\
& \mathcal{B}(\eta)=g_{m, n}^{-1}\left[h_{m, n}^{-1}\left(\mathcal{A}_{1}^{(m-1) /(m+1)}\left(\eta-\eta_{0}\right)\right)\right],
\end{aligned}
$$

where

$$
h_{m, n}(v) \equiv \int_{v_{0}}^{v} \frac{\mathrm{d} s}{1-g_{m, n}^{-1}(s)} .
$$

The precise location of the reaction front is set by choosing $v\left(s_{0}\right)=v_{0}>0$ for some constant $s_{0}=\mathcal{A}_{1}^{(m-1) /(m+1)} \eta_{0}$. With these results, the inner problem is reduced to the solution of two algebraic equations for $g_{m, n}^{-1}(v)$ and $h_{m, n}^{-1}(\eta)$ and one quadrature (59). In practice, however, it is simpler to integrate (49) directly.

Numerical solutions of the rescaled inner problem

$$
v^{\prime \prime}=v^{m}\left(1-v^{\prime}\right)^{n}, \quad v(-\infty)=0, \quad v^{\prime}(\infty)=1
$$

are obtained by a shooting method with the results shown in Fig. 5(a) for $m=n=1$ and $m=n=2$. The position of the front is chosen such that $v(5)=5$. The static reactant concentration $u(s)$ and the reaction rate density $v^{m} u^{n}$ are shown in Figs. 5(b) and (c), respectively. Note that the concentration fields decay to their asymptotic values as $|\eta| \rightarrow \infty$ more slowly as $m$ and $n$ are increased above unity, a phenomenon that we explore analytically in the next section. 

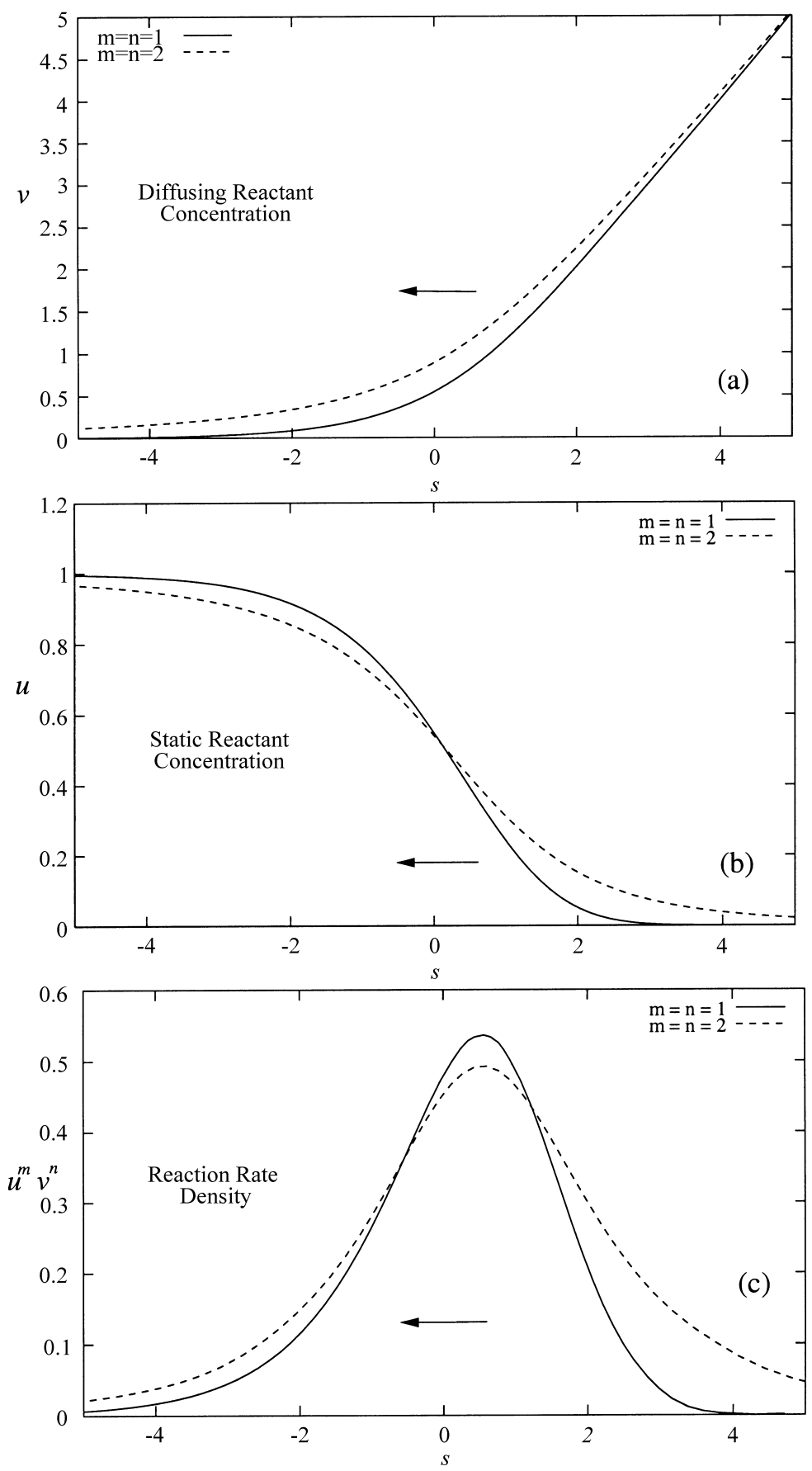

Fig. 5. Structure of the reaction front obtained by numerical solutions of Eq. (60) with $s_{0}$ chosen to set $v(5)=5$. Profiles of (a) $v(s)=\mathcal{A}(\eta) \mathcal{A}_{1}^{-2 /(m+1)}$, (b) the static reactant concentration $u(s)=\mathcal{B}(\eta)$ and (c) the reaction rate density $u(s)^{m} v(s)^{n}=\mathcal{R}(\eta) \mathcal{A}_{1}^{-2 m /(m+1)}$ versus $s=\eta \mathcal{A}_{1}^{(m-1) /(1+m)}$ are shown for $m=n=1$ and $m=n=2$. Arrows indicate that the reaction front propagates from right to left. 


\subsection{Localization of the reaction front}

The width of the reaction front varies in time according to $w(t) \sim t^{\alpha}$, where $\alpha=(m-1) / 2(m+1)$. On the scale of the diffusion layer width $W(t) \sim t^{1 / 2}$, the reaction front is "localized" after long times because $\alpha<\frac{1}{2}$. Note that the overall localization of the front $w(t) / W(t)$ is controlled by $m$ (the reaction order of the diffusing species A), but we now show that both $m$ and $n$ (the reaction order of the static species B) affect localization on the scale of $w(t)$. Specifically, we derive the spatial decay of the scaled reaction rate $\mathcal{R}(\eta)=\mathcal{A}(\eta)^{m} \mathcal{B}(\eta)^{n}$ in terms of the inner similarity variable $\eta \rightarrow \pm \infty$ (see Fig. 5(c)). The actual reaction rate decays uniformly to zero in time, $r \sim t^{-\beta} \mathcal{R}(\eta)$ with $\beta=m /(m+1)$, but here we are only concerned with the shape of $\mathcal{R}(\eta)$.

Ahead of the reaction front in the limit $\eta \rightarrow-\infty$, we have $\mathcal{A}^{\prime \prime} \sim \mathcal{A}^{m}$ from (49), which can be integrated to obtain the decay of concentration fields:

$$
\begin{aligned}
& \mathcal{A}(\eta) \sim \begin{cases}\mathcal{A}_{2} \mathrm{e}^{-|\eta|} & \text { if } m=1, \\
\mathcal{A}_{2}|\eta|^{-2 /(m-1)} & \text { if } m>1,\end{cases} \\
& 1-\mathcal{B}(\eta) \sim \begin{cases}\mathcal{A}_{3} \mathrm{e}^{-|\eta|} & \text { if } m=1, \\
\mathcal{A}_{3}|\eta|^{-(m+1) /(m-1)} & \text { if } m>1,\end{cases} \\
& \mathcal{R}(\eta) \sim \begin{cases}\mathcal{A}_{2}^{m} \mathrm{e}^{-m|\eta|} & \text { if } m=1, \\
\mathcal{A}_{2}^{m}|\eta|^{-2 m /(m-1)} & \text { if } m>1,\end{cases}
\end{aligned}
$$

where $\mathcal{A}_{2}$ and $\mathcal{A}_{3}$ are constants. Note that the localization of $\mathcal{A}(\eta)$ and $\mathcal{R}(\eta)$ ahead of the front is entirely controlled by the reaction order $m$ of the depleted reactant (which is the diffusing species A). There is a transition from an exponential decay for $m=1$ to a slower power-law decay for $m>1$.

Next we consider localization of $\mathcal{R}(\eta)$ behind the reaction front in the limit $\eta \rightarrow \infty$. From asymptotic matching with the diffusion layer, we have already derived $\mathcal{A}(\eta) \sim \mathcal{A}_{1} \eta$. The asymptotic decay of $\mathcal{B}(\eta)$ and $\mathcal{R}(\eta)$ is obtained by integrating $\mathcal{A}_{1} \mathcal{B}^{\prime} \sim-\left(\mathcal{A}_{1} \eta\right)^{m} \mathcal{B}^{n}$ :

$$
\begin{aligned}
& \mathcal{B}(\eta) \sim \begin{cases}\mathcal{B}_{1} \exp \left[-\mathcal{A}_{1}^{m-1} \eta^{m+1} /(m+1)\right] & \text { if } n=1, \\
\mathcal{B}_{1} \eta^{-(m+1) /(n-1)} & \text { if } n>1,\end{cases} \\
& \mathcal{R}(\eta) \sim \begin{cases}\mathcal{A}_{1}^{m} \mathcal{B}_{1}^{n} \eta^{m} \exp \left[-\mathcal{A}_{1}^{m-1} \eta^{m+1} /(m+1)\right] & \text { if } n=1, \\
\mathcal{A}_{1}^{m} \mathcal{B}_{1}^{n} \eta^{-(m+n) /(n-1)} & \text { if } n>1,\end{cases}
\end{aligned}
$$

where $\mathcal{B}_{1}$ is a constant. Once again, a higher reaction order $n$ for the depleted species (which is the static species B) broadens the front: there is another transition from exponential decay for $n=1$ to a power-law decay for $n>1$. Note, however, that increasing the reaction order $m$ of the diffusing species A contracts the back side of the front.

The fact that $\mathcal{R}(\eta)$ has a fairly broad, power-law decay for $n>1$ toward the diffusion layer should cause concern since we have previously assumed in (28) that the reaction term is negligible in the diffusion layer. Fortunately, however, for all $m, n \geq 1$, the decay of $\mathcal{B}(\eta)$ is just fast enough to satisfy (28) in the intermediate region where $B(0<\zeta \ll 1) \approx \mathcal{B}(\eta \gg 1)$. From (62a) along with $\tilde{A}(\zeta, t) \sim A(\zeta), \tilde{\mathcal{B}}(\eta, t) \sim \mathcal{B}(\eta), \eta=2 \zeta t^{1 /(m+1)}$ and $\delta=\frac{1}{2}$, we have

$$
t^{2 \delta} \tilde{A}(\zeta, t)^{m} \tilde{B}(\zeta, t)^{n}= \begin{cases}\mathrm{O}\left(t \exp \left[-\mathcal{A}_{1}^{m-1}(2 \zeta)^{m+1} t /(m+1)\right]\right) & \text { if } n=1, \\ \mathrm{O}\left(\zeta^{-n(m+1) /(n-1)} t^{-n /(n-1)}\right) & \text { if } n>1,\end{cases}
$$

as $t \rightarrow \infty$ with $0<\zeta \ll 1$ fixed, which verifies (28) in the intermediate region for any $m, n \geq 1$. However, we now prove that (28) actually holds throughout the diffusion layer for all $\zeta>0$. 


\section{Transient decay in the diffusion layer}

\subsection{Assumption of quasi-stationarity}

The analysis of long-time asymptotics in the previous section rests on two basic assumptions:

1. "scale separation", given by

$$
\begin{aligned}
& 0<\lim _{t \rightarrow \infty} \tilde{\mathcal{A}}(\eta, t)^{m} \tilde{B}(\zeta, t)^{n}<\infty \text { for }|\eta|<\infty, \\
& \lim _{t \rightarrow \infty} t \tilde{A}(\zeta, t)^{m} \tilde{B}(\zeta, t)^{n}=0 \text { for } \zeta>0,
\end{aligned}
$$

2. "quasi-stationarity", given by (20a), (20b), (26a) and (26b) along with

$$
\begin{aligned}
& \lim _{t \rightarrow \infty} t^{(m-1) /(m+1)} \frac{\partial \tilde{\mathcal{A}}}{\partial t}(\eta, t)=0 \quad \text { for }|\eta|<\infty, \\
& \lim _{t \rightarrow \infty} t^{m /(m+1)} \frac{\partial \tilde{\mathcal{B}}}{\partial t}(\eta, t)=0 \text { for }|\eta|<\infty, \\
& \lim _{t \rightarrow \infty} t \frac{\partial \tilde{A}}{\partial t}(\zeta, t)=0 \quad \text { for } \zeta>0 .
\end{aligned}
$$

Assumption 1 states that two spatial regions with disparate length scales, the reaction front and the diffusion layer, arise where the reaction term on the right-hand side of the governing partial integro-differential equation (14) or (15) is, respectively, either comparable to or dominated by the diffusion term. Assumption 2 states that, when viewed on scales appropriate for each region, the solution to the initial-boundary-value problem (10a) and (10b) approaches an asymptotically self-similar form, which is suggested by the fact that there is no natural length scale in the problem.

These ubiquitous assumptions $[1,13,14,50]$ have been rigorously justified $[11,12]$ in the special case of a perfectly symmetric $\left(D_{\mathrm{A}}=D_{\mathrm{B}}, \rho_{\mathrm{A}}^{0}=\rho_{\mathrm{B}}^{0}\right)$, and thus stationary $(\nu=0)$, reaction front involving two diffusing reactants with certain kinetic orders ( $m=n=1$ and $m=n>3$ ). To our knowledge, a similar mathematical validation of these assumptions has not been given for the general situation of a moving reaction front with arbitrary kinetic orders for either one or two diffusing reactants. For one static reactant, however, convergence at the diffusive scale has been rigorously established by Hilhorst et al. [15], even with a very general reaction term [16].

In this section, we prove the more modest result that quasi-stationarity implies scale separation, i.e. (65a)-(65c) implies (64a) and (64b). (It suffices to show (64b) since (64a) and (64b) follows from the definition of $\eta$ in Section 3.1.) Although this analysis justifies a posteriori the assumption of scale separation in our fairly general situation $\left(D_{\mathrm{B}}=0, v>0, m, n \geq 1\right)$, it more importantly reveals the transient decay to the asymptotic similarity solution in the diffusion layer. Specifically, we derive exact formulae for the asymptotic decay of the reaction-rate density and static-reactant concentration in the diffusion layer.

We begin by precisely stating our assumptions related to (65a)-(65c). From (65a) and its consequence (61a), we conclude that the diffusing reactant concentration vanishes on the scale $W(t)=\sqrt{t}$ ahead of the front (where there is no diffusion layer) since $\tilde{A}(\zeta, t) \sim \mathcal{A}\left(2 \zeta t^{1 /(m+1)}\right) \rightarrow 0$ as $t \rightarrow \infty$ with $\zeta<0$ fixed. This result can be combined with (26a) to obtain a statement of quasi-stationarity on the scale $W(t)$ :

$$
\tilde{A}(\zeta, t) \rightarrow A(\zeta) H(\zeta) \text { and } \quad \frac{\partial \tilde{A}}{\partial \zeta}(\zeta, t) \rightarrow A^{\prime}(\zeta) H(\zeta)
$$

as $t \rightarrow \infty$ with $\zeta \neq 0$ fixed. We have already derived the exact form of the similarity function $A(\zeta)$ in $(35)$ as a consequence of neglecting reactions (64b). To avoid a circular argument, however, we must now establish (64b) 
without using (35), thus giving a posteriori justification for the latter equation. Throughout Section 4, our only assumptions about $A(\zeta)$ are $A(0)=0$ and $A^{\prime}(0)>0$. These properties follow from matching with the reaction front, where $a \rightarrow 0$ (i.e. $\gamma>0$ ) follows from quasi-stationarity, as shown in Section 3.1.

\subsection{Direction of the diffusing-reactant flux}

Let us prove that $A(\zeta)$ is strictly increasing in the diffusion layer, $A^{\prime}(\zeta)>0$ for all $\zeta \geq 0$, as a consequence of (64a). Combining (65c) with (25a), we have

$$
-2(\zeta-v) \frac{\partial \tilde{A}}{\partial \zeta} \sim \frac{\partial^{2} \tilde{A}}{\partial \zeta^{2}}-t \tilde{A}^{m} \tilde{B}^{n} \quad \text { as } t \rightarrow \infty \text { with } \zeta>0 \text { fixed, }
$$

which is easily integrated once using an integrating factor,

$$
\frac{\partial \tilde{A}}{\partial \zeta}(\zeta, t) \sim \mathrm{e}^{-(\zeta-v)^{2}}\left[\mathrm{e}^{\nu^{2}} \frac{\partial \tilde{A}}{\partial \zeta}(0, t)+t \int_{0}^{\zeta} \tilde{A}(\xi, t)^{m} \tilde{B}(\xi, t)^{n} \mathrm{e}^{(\xi-v)^{2}} \mathrm{~d} \xi\right]
$$

Since the second term on the right-hand side is non-negative for all $t>0$, we can pass to the limit $t \rightarrow \infty$ for any fixed $\zeta>0$ to obtain the desired bound

$$
A^{\prime}(\zeta) \geq A^{\prime}(0) \exp \left[v^{2}-(\zeta-v)^{2}\right]>0,
$$

which expresses the physical fact that everywhere in the diffusion layer, a nonzero flux of the diffusing species is directed toward the reaction front (at sufficiently large times).

\subsection{Decay of the static-reactant concentration}

We now prove that $b(x, t)$ vanishes asymptotically in the diffusion layer as a consequence of quasi-stationarity, which implies

$$
\tilde{B}(\zeta, t) \rightarrow B(\zeta)=H(-\zeta) \quad \text { as } t \rightarrow \infty \text { with } \zeta \neq 0 \text { fixed. }
$$

For $\zeta<0$, this follows from (61b) since there is no diffusion layer ahead of the front, and therefore $\tilde{B}(\zeta, t) \sim$ $\mathcal{B}\left(2 \zeta t^{1 /(m+1)}\right) \rightarrow 1=B(\zeta)$ as $t \rightarrow \infty$ with $\zeta<0$ fixed. Likewise, in Section 2.1, we have already established (70) for $\zeta>v$ since $\tilde{B}(\zeta, t)$ vanishes there identically for all times. Therefore, it only remains to prove that $B(\zeta)=0$ for $0<\zeta<v$.

In light of the expression for $b(x, t)$ in (12), the definition of $\phi_{m}(x, t)$ in (13) and the restriction $n \geq 1$, it suffices to show that $\Phi_{m}(\zeta, t) \equiv \phi_{m}(x, t) \rightarrow \infty$ as $t \rightarrow \infty$ for $0<\zeta<v$ fixed. Using $\zeta=v+x / 2 \sqrt{t}$ (since $\delta=\sigma=\frac{1}{2}$ ), we transform $\phi_{m}(x, t)$ into the diffusion-layer coordinates $(x, t) \mapsto(\zeta, t)$ :

$$
\Phi_{m}(\zeta, t)=\int_{0}^{t} a(2 \sqrt{t}(\zeta-v), \tau)^{m} \mathrm{~d} \tau
$$

and express this in terms of the diffusion-layer scaling function $a(x, \tau)=\tilde{A}(\nu+x / 2 \sqrt{\tau}, \tau)$ :

$$
\Phi_{m}(\zeta, t)=\int_{0}^{t} \tilde{A}\left(\sqrt{\frac{t}{\tau}}(\zeta-v)+v, \tau\right)^{m} \mathrm{~d} \tau
$$


It is convenient to work with the partial time-derivative of $\Phi_{m}(\zeta, t)$ given by the Leibniz rule:

$$
\frac{\partial \Phi_{m}}{\partial t}=\tilde{A}(\zeta, t)^{m}+\int_{0}^{t} \frac{\partial \tilde{A}^{m}}{\partial \zeta}\left(\sqrt{\frac{t}{\tau}}(\zeta-v)+\nu, \tau\right) \frac{1}{2 t} \sqrt{\frac{t}{\tau}}(\zeta-v) \mathrm{d} \tau .
$$

Focusing on the region $0<\zeta<v$, we make the transformation $\xi=\sqrt{t / \tau}(\zeta-v)+v$,

$$
\frac{\partial \Phi_{m}}{\partial t}=\tilde{A}(\zeta, t)^{m}-\int_{-\infty}^{\zeta} \frac{\partial \tilde{A}^{m}}{\partial \zeta}\left(\xi,\left(\frac{\zeta-v}{\xi-v}\right)^{2} t\right)\left(\frac{\zeta-v}{\xi-v}\right)^{2} \mathrm{~d} \xi,
$$

and pass the limit $t \rightarrow \infty$ inside the integral to obtain

$$
\lim _{t \rightarrow \infty} \frac{\partial \Phi_{m}}{\partial t}=A(\zeta)^{m}-\int_{0}^{\zeta} \frac{\mathrm{d} A^{m}}{\mathrm{~d} \zeta}(\xi)\left(\frac{\zeta-v}{\xi-v}\right)^{2} \mathrm{~d} \xi
$$

where the lower limit of integration follows from (66) since $A(\zeta)=A^{\prime}(\zeta)=0$ for $\zeta<0$. This step is justified by the dominated convergence theorem [31] because, by virtue of (66), there exist constants $M, t_{0}>0$ such that the integrand in (74) is bounded for all $t>t_{0}$ by $M /(\xi-v)^{2}$, which is integrable on $(-\infty, \zeta)$ if $\zeta<v$.

Since $A(0)=0$ is required by matching between the two regions of quasi-stationarity, Eq. (75) can be written in the form $\partial \Phi_{m} / \partial t \sim f_{m}(\zeta)$, where

$$
f_{m}(\zeta) \equiv \int_{0}^{\zeta} \frac{\mathrm{d} A^{m}}{\mathrm{~d} \zeta}(\xi)\left[1-\left(\frac{\zeta-v}{\xi-v}\right)^{2}\right] \mathrm{d} \xi
$$

Note that $f_{m}(\zeta)>0$ for $0<\zeta<v$ since $A^{\prime}(\zeta)>0$ in this region, as shown in (69). Therefore, with an integration of (76), we arrive at the desired result

$$
\Phi_{m}(\zeta) \sim f_{m}(\zeta) t \quad \text { as } t \rightarrow \infty \text { with } 0<\zeta<v \text { fixed }
$$

thus completing the proof that $B(\zeta)=0$ for $\zeta>0$.

By substituting (77) into (12), we obtain the transient decay of $\tilde{B}(\zeta, t)$ in part of the diffusion layer where the reaction front has already passed $(0<\zeta \leq v)$ :

$$
\tilde{B}(\zeta, t) \sim \begin{cases}\mathrm{e}^{-q f_{m}(\zeta) t} & \text { if } n=1 \\ {\left[q(n-1) f_{m}(\zeta) t\right]^{-1 /(n-1)}} & \text { if } n>1\end{cases}
$$

Note that $\tilde{B}(\zeta, t)$ vanishes with exponential decay if $n=1$ and with a power-law decay if $n>1$. Therefore, by measuring the asymptotic decay (either exponential or power-law) of the static-reactant concentration in the diffusion layer, the reaction order $n$ could in principle be inferred from experimental data (although such measurements are difficult in practice [3]).

\subsection{Decay of the reaction-rate density}

From (78) we easily obtain the asymptotic decay on the reaction rate density in the diffusion layer as $t \rightarrow \infty$ with $\zeta>0$ fixed:

$$
t \tilde{A}(\zeta, t)^{m} \tilde{B}(\zeta, t)^{n} \sim \begin{cases}A(\zeta)^{m} t \mathrm{e}^{-q f_{m}(\zeta) t} & \text { if } n=1, \\ A(\zeta)^{m}\left[q(n-1) f_{m}(\zeta)\right]^{-n /(n-1)} t^{-1 /(n-1)} & \text { if } n>1,\end{cases}
$$

which establishes (64b). The reaction term in the diffusion layer has previously been neglected based only on physical intuition [14], but here we have given a mathematical justification. 


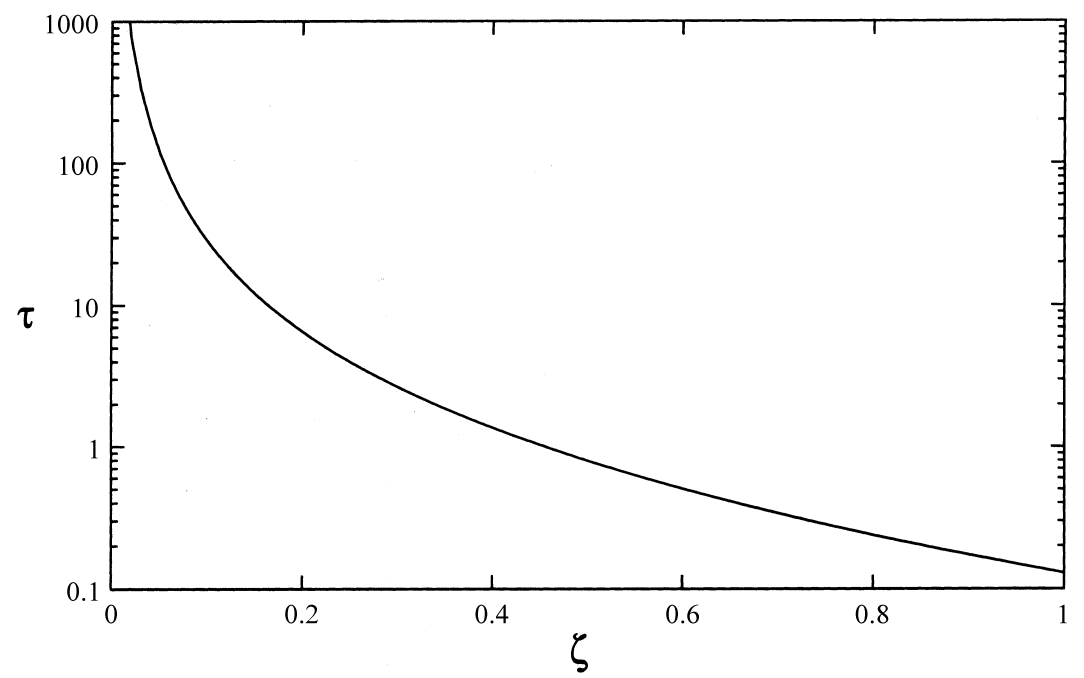

Fig. 6. Transient decay time $\tau_{1}(\zeta)$ given by (80) for the reaction rate and static-reactant concentration in the diffusion layer when $m=n=1$.

\subsection{The decay time when $n=1$}

Since the reaction term vanishes sufficiently fast in the diffusion layer to justify a posteriori the analysis in Section 3, the exact expression for $A(\zeta)$ from (35) may be substituted into (76) to evaluate the function $f_{m}(\zeta)$. If $n>1$, then $f_{m}(\zeta)$ affects the transient decay in (78) and (79) only as a multiplicative prefactor in a power law, which would be difficult to measure in a real experiment. If $n=1$, however, then $f_{m}(\zeta)$ sets the characteristic time $\tau_{m}(\zeta)^{-1} \equiv q f_{m}(\zeta)$ of an exponential decay, which is easier to measure experimentally. Therefore, we now derive an exact expression for the decay time $\tau_{1}(\zeta)(0<\zeta \leq \nu)$ in the case $m=1$ :

$$
\begin{aligned}
\tau_{1}(\zeta)^{-1} & =q f_{1}(\zeta)=\int_{0}^{\zeta} 2 v \mathrm{e}^{v^{2}-(v-\xi)^{2}}\left[1-\left(\frac{\zeta-v}{\xi-v}\right)^{2}\right] \mathrm{d} \xi \\
& =2(v-\zeta)\left[(v-\zeta)-v \mathrm{e}^{v^{2}-(v-\zeta)^{2}}\right]+\sqrt{\pi} v \mathrm{e}^{v^{2}}\left[1+2(v-\zeta)^{2}\right][\operatorname{erf}(v)-\operatorname{erf}(v-\zeta)] .
\end{aligned}
$$

Note that $\tau_{1}(0)=\infty$ in the vicinity of the reaction front $(\zeta=0)$ because (78) and (79) no longer hold. Within the diffusion layer, the decay time is a decreasing function of distance $\zeta$ away from the reaction front, as shown in Fig. 6. These results may be used to infer reaction orders and perhaps even kinetic constants in diffusion-limited corrosion experiments from transient decay measurements of the reaction-rate density in the diffusion layer [3].

\section{Uniformly valid asymptotic approximations}

In the previous two sections we have argued for the existence of a unique asymptotic similarity solution (up to an unknown constant $\eta_{0}$ ) contingent upon certain "quasi-stationarity" conditions, which are likely to be satisfied for the specified initial conditions (see below). This solution, valid after long times, consists of two different asymptotic approximations for $a(x, t)$, the concentration of the diffusing reactant $\mathrm{A}$, which reflect the different couplings of length and time in the reaction front and the diffusion layer. A single asymptotic approximation for $a(x, t)$ that 
is uniformly valid across all space is obtained by adding the two contributions from the reaction front (the inner region) and the diffusion layer (the outer region) and subtracting the overlap (from the intermediate region) [27,28]:

$$
a(x, t) \sim\left[\mathcal{A}\left(\eta-\eta_{0}\right)-\mathcal{A}_{1}\left(\eta-\eta_{0}\right) H\left(\eta-\eta_{0}\right)\right] t^{-1 /(m+1)}+A(\zeta) H(\zeta) \quad \text { as } t \rightarrow \infty \text { for all } x,
$$

where the reaction-front and diffusion-layer similarity variables are

$$
\begin{aligned}
& \eta(x, t)=\frac{x+2 v t^{1 / 2}}{t^{(m-1) / 2(m+1)}}, \\
& \zeta(x, t)=\frac{x+2 v t^{1 / 2}}{2 t^{1 / 2}},
\end{aligned}
$$

$v(q)^{2}$ is the diffusion constant of the reaction front (see (44) and Fig. 3), $\mathcal{A}_{1}=v(q) / q$ is a constant proportional to the diffusive flux entering the front, $\mathcal{A}(\eta)$ is the reaction-front similarity function (see (58a) and Fig. 5), $A(\zeta)$ is the diffusion-layer similarity function (see (35) and Fig. 2) and $\eta_{0}$ is an undetermined constant depending upon the initial conditions as well as the precise definition of the reaction-front location. The uniform approximation has been determined analytically up to the solution of two algebraic equations (58a) and (58b) and one quadrature (59).

A subtle point in the construction of this uniformly valid approximation is that shifting the position of the front by $\eta \mapsto \eta-\eta_{0}$ does not affect matching with the diffusion layer because in that case $\zeta \mapsto \frac{1}{2}\left(\eta-\eta_{0}\right) t^{-1 /(m+1)} \sim$ $\frac{1}{2} \eta t^{-1 /(m+1)}=\zeta$. In other words, because the reaction front is "infinitely thin" compared to the diffusion layer, translating its similarity variable by a constant $\eta_{0}$, or any other function of time that is o $\left(t^{1 / 2}\right)$, does not require that the diffusion-layer similarity variable $\zeta$ be shifted as well.

The situation for $b(x, t)$, the concentration of the static reactant $\mathrm{B}$, is much simpler. By comparing the asymptotic bound on $b(x, t)$ in the diffusion layer given by (78) with the tail of the reaction-front approximation given by (62a) with $\eta=2 \zeta t^{1 /(m+1)}$, we see that the asymptotic behavior of $b(x, t)$ is identical in the two regions. Therefore,

$$
b(x, t) \sim \mathcal{B}\left(\eta-\eta_{0}\right) \quad \text { as } t \rightarrow \infty \text { for all } x
$$

is a uniformly valid approximation, where $\mathcal{B}(\eta)$ is the reaction-front similarity function given by $(58 \mathrm{~b})$.

At this point, the initial conditions have not yet entered the analysis except in (12), which only influences the prefactors of the transient-decay formulae in Section 4. Therefore, the asymptotic similarity solution is universal up to a constant shift of the reaction front by $\eta_{0}$ for some broad set of initial conditions which presumably contains (10d). In general, this "universality class" of initial conditions leading to the same asymptotic similarity solution (up to different values of $\eta_{0}$ ) is expected to be attained whenever the initial reaction-rate distribution $r(x, 0)=$ $a(x, 0)^{m} b(x, 0)^{n}$ is sufficiently well localized and the reactants are sufficiently well separated. This class surely contains all initial conditions for which $r(x, 0)$ has compact support, e.g. $r(x, 0)=0$ for $x \neq 0$ in $(10 \mathrm{~d})$, or exponential decay, e.g. $r(x, 0)<M \mathrm{e}^{-|x| / x_{0}}$ for some $M, x_{0}>0$, but perhaps not slower power-law decay.

\section{Discussion}

In this article we have studied the long-time asymptotics of solutions to the initial-boundary-value problem of (3)-(6), which is a generic mean-field model for the corrosion of a porous solid by a diffusing chemical. We have derived a uniformly valid asymptotic approximation (81) and (83) consisting of matched similarity solutions in two distinct regions, the reaction front and diffusion layer, each possessing different power-law scaling behavior. The existence and uniqueness of the similarity functions and the scaling exponents have been established if and only if $m, n \geq 1$, and through an analysis of transients in the diffusion layer the asymptotic scale separation has been shown 
Table 1

Comparison of the two possible sets of scaling exponents for asymptotic similarity solutions to the one-dimensional, mean-field reaction-diffusion equations for two initially separated reactants (the width of the reaction front is given by $w \sim t^{\alpha}$, the magnitude of the reaction rate by $R \sim t^{-\beta}$ and the concentration of a diffusing species in the reaction front by $a \sim t^{-\gamma}$ )

\begin{tabular}{lccc}
\hline & $\alpha$ & $\beta$ & $\gamma$ \\
\hline (i) $D_{\mathrm{A}}>0, D_{\mathrm{B}}=0$ & $\frac{m-1}{2(m+1)}$ & $\frac{m}{m+1}$ & 1 \\
(ii) $D_{\mathrm{A}}>0, D_{\mathrm{B}}>0$ & $\frac{m+n-1}{m+1}$ & $\frac{m+n}{m+n+1}$ & 1 \\
\hline
\end{tabular}

to follow from the assumption of quasi-stationarity. Since quasi-stationarity has been observed in recent experiments on the corrosion of ramified electrodeposits [3], the present analysis therefore suffices to establish the theoretical predictions of the mean-field equations for at least one particular corrosion system. Although the case considered here ( $m, n \geq 1, q \neq 1$ ) is more complicated, it would be useful to perform a rigorous transient analysis along the lines of Schenkel et al. [11] (who considered only the case $D_{\mathrm{A}}=D_{\mathrm{B}} \neq 0, q=1$ and $m=n=1$ ). Nevertheless, we have at least provided a firm mathematical justification for the scale separation between the diffusion layer and reaction front.

In this work we have paid special attention to the effect of higher-order reactions $(m, n>1)$. First of all, the scaling exponents vary with the reaction order $m$ of the diffusing reactant in precisely the same way as they do on the sum $m+n$ in the case of two diffusing reactants [5,44-47], as shown in Table 1. Moreover, the spatial localization of the reaction rate $r(a, b)$ on each side of the front depends primarily on the reaction order of the depleted reactant: as the appropriate reaction order is increased from unity, the spatial dependence of the reaction rate away from the front changes from an exponential decay to a progressively broader power-law decay. Similarly, the temporal decay of the depleted (static) reactant concentration in the diffusion layer depends sensitively on its reaction order, undergoing a transition from exponential to power-law decay (in time) as $n$ is increased from unity. These properties may have general relevance for more complicated multi-component reaction-diffusion systems.

Other qualitiative features of our analysis that might have more general applicability are the dominant balances in the reaction front, where the concentration of a diffusing reactant is determined by a balance between reactions and "steady state" diffusion, i.e. a mobile species diffuses slowly to the front where it immediately reacts. On the other hand, the concentration of a static reactant is determined by a balance between reactions and fictitous advection due to the moving reference frame, i.e. since the static species cannot diffuse to the front, the front must diffuse to it. These guiding principles might help simplify more complicated reaction-diffusion equations for which asymptotically self-similar solutions do not exist.

\section{Epilogue}

After the writing of this article, a referee pointed out some interesting similarities between our analysis of chemical reaction fronts and various existing studies in combustion theory [32,33]. Indeed, the equations for combustion waves introduced by Zeldovich and Frank-Kamenetzki [32], which have since been generalized by many authors (e.g. [34]), bear some resemblance to (2a) and (2b) since they describe the diffusion of a fuel substance coupled to the diffusion of heat. In combustion theory, however, the usual reaction term is quite different from (5) because it involves exponential Arrhenius temperature dependence, and the initial and boundary conditions also differ from those considered here. As a result, combustion waves tend to exhibit qualitatively different behavior from reaction-diffusion fronts. (For example, simple flame fronts have constant width and constant velocity.) Nevertheless, combustion waves exhibit 
multiple scales analogous to the reaction-front and diffusion layers described here, which have also been analyzed using matched asymptotic expansions [35] (although not in the dynamical setting of this work). The idea of matching derivatives between the inner and outer regions actually appears to have its origin in the pioneering paper of Zeldovich and Frank-Kamenetzki [32], in which the velocity of a simple flame front is determined by analyzing a single-component equation like (8). In hindsight, it is somewhat surprising that the recent parallel literatures on two-species reaction-diffusion fronts and combustion waves have developed quite independent of each other without any cross-references (at least, none to our knowledge). It is hoped, therefore, that this paper will initiate the "diffusion" of ideas between these two mature but related disciplines.

\section{Acknowledgements}

The authors thank C. Léger and R.R. Rosales for useful discussions. This work was supported by an NSF infrastructure grant (MZB) and grants from the Harvard MRSEC DMR-980-9363 and the Army Research Office DAAG-55-97-1-0114 (HAS).

\section{References}

[1] L. Gálfi, Z. Rácz, Phys. Rev. A 38 (1988) 3151.

[2] Y.-E. Koo, L. Li, R. Kopelman, Mol. Cryst. Liq. Cryst. 183 (1990) 187.

[3] C. Léger, F. Argoul, M.Z. Bazant, J. Phys. Chem. B 103 (1999) 5841 (http://xxx.lanl.gov/abs/physics/9902006).

[4] Z. Jiang, C. Ebner, Phys. Rev. A 42 (1990) 7483.

[5] B. Chopard, M. Droz, Europhys. Lett. 15 (1991) 45.

[6] S. Cornell, M. Droz, Physica D 103 (1997) 348.

[7] M. Araujo, S. Havlin, H. Larralde, H.E. Stanley, Phys. Rev. Lett. 68 (1992) 1791.

[8] S. Havlin, M. Araujo, Y. Lereah, H. Larralde, A. Shehter, H.E. Stanley, P. Trunfio, B. Vilensky, Physica A 221 (1995) 1.

[9] Z. Koza, H. Taitelbaum, Phys. Rev. E 54 (1996) R1040.

[10] H. Taitelbaum, A. Yen, R. Kopelman, S. Havlin, G.H. Weiss, Phys. Rev. E 54 (1996) 5942.

[11] A. Schenkel, P. Wittwer, J. Stubbe, Physica D 69 (1993) 135.

[12] G. van Baalen, A. Schenkel, P. Wittwer, Commun. Math. Phys. 210 (2000) 145.

[13] Z. Koza, J. Stat. Phys. 85 (1996) 179.

[14] Z. Koza, Physica A 240 (1997) 622.

[15] D. Hilhorst, R. van der Hout, L.A. Peletier, J. Math. Anal. Appl. 199 (1996) 349.

[16] D. Hilhorst, R. van der Hout, L.A. Peletier, Preprints, 1999.

[17] P. Grindrod, Theory and Applications of Reaction-Diffusion Equations, Clarendon Press, Oxford, 1996.

[18] R.A. Alberty, Physical Chemistry, 6th Edition, Wiley, New York, 1983.

[19] J.D. Murray, Lectures on Non-linear Differential Equation Models in Biology, Clarendon Press, Oxford, 1977.

[20] J.D. Murray, Mathematical Biology, 2nd Edition, Springer, New York, 1993.

[21] B. Lee, J. Cardy, Phys. Rev. E 50 (1994) 3287.

[22] D.G. Aronson, H.F. Weinberger, Nonlinear Diffusion in Population Genetics, Combustion and Nerve Pulse Propagation, Lecture Notes in Mathematics, Vol. 446, Springer, New York, 1975, p. 12.

[23] A. Gmira, L. Veron, J. Differential Equations 53 (1984) 258.

[24] G.I. Barenblatt, Similarity, Self-Similarity and Intermediate Asymptotics, 2nd Edition, Cambridge University Press, Cambridge, MA, 1996.

[25] G.I. Barenblatt, Dimensional Analysis, Gordon and Breach, New York, 1987.

[26] L. Dresner, Similarity Solutions of Nonlinear Partial Differential Equations, Research Notes in Mathematics, Vol. 88, Pitman, London, 1983.

[27] E.J. Hinch, Perturbation Methods, Cambridge University Press, Cambridge, MA, 1991.

[28] C.M. Bender, S.A. Orszag, Advanced Mathematical Methods for Scientists and Engineers, McGraw-Hill, New York, 1978.

[29] H.T. Davis, Introduction to Nonlinear Differential and Integral Equations, Dover, New York, 1962.

[30] M. Abramowitz, I.A. Stegun (Eds.), Handbook of Mathematical Functions, Dover, New York, 1965.

[31] L.C. Evans, R. Gariepy, Measure Theory and Fine Properties of Functions, CRC Press, Boca Raton, FL, 1992.

[32] Ya.B. Zeldovich (originally translated as J.B. Zeldowitch), D.A. Frank-Kamenetzki, Acta Physicochim. URSS 9 (1938) 341. 
[33] Ya.B. Zeldovich, G.I. Barenblatt, V.B. Librovich, G.M. Makhviladze, Mathematical Theory of Combustion and Explosion, Consultants Bureau, New York, 1985.

[34] B.J. Matkowsky, G.I. Sivashinsky, SIAM J. Appl. Math. 37 (1979) 686.

[35] D. Schult, SIAM J. Appl. Math. 60 (1999) 136.

[36] Y.-E. Koo, R. Kopelman, J. Stat. Phys. 65 (1991) 893.

[37] H. Taitelbaum, Y.-E. Koo, S. Havlin, R. Kopelman, G. Weiss, Phys. Rev. A 46 (1992) 2151.

[38] H. Taitelbaum, B. Vilensky, A. Lin, Y.-E. Koo, R. Kopelman, Phys. Rev. Lett. 77 (1996) 1640.

[39] H. Taitelbaum, A. Yen, R. Kopelman, S. Havlin, G. Weiss, Phys. Rev. E 54 (1996) 5942.

[40] A. Yen, Y.-E. Koo, R. Kopelman, Phys. Rev. E 54 (1996) 2447.

[41] A. Yen, A. Lin, Y.-E. Koo, B. Vilensky, H. Taitelbaum, R. Kopelman, J. Phys. Chem. A 101 (1997) 2819.

[42] A. Yen, R. Kopelman, Phys. Rev. E 56 (1997) 3694.

[43] A. Yen, Z.-Y. Shi, R. Kopelman, Phys. Rev. E 57 (1998) 2438.

[44] S. Cornell, M. Droz, B. Chopard, Phys. Rev. A 44 (1991) 4826.

[45] S. Cornell, M. Droz, B. Chopard, Physica A 188 (1992) 322.

[46] S. Cornell, M. Droz, Phys. Rev. Lett. 70 (1993) 3284.

[47] S. Cornell, Z. Koza, M. Droz, Phys. Rev. E 52 (1995) 3500.

[48] M. Araujo, H. Larralde, S. Havlin, H.E. Stanley, Phys. Rev. Lett. 71 (1993) 3592.

[49] H. Taitelbaum, Z. Koza, Phil. Mag. B 77 (1998) 1389.

[50] Z. Koza, Phil. Mag. B 77 (1998) 1437.

[51] M. Howard, J. Cardy, J. Phys. A 28 (1995) 3599. 